\title{
Updated checklist of orchid flora of Manipur
}

\author{
A. N. Rao' ${ }^{1}$ V. Kumar ${ }^{2}$ \\ ${ }^{1}$ Orchid Research and Development Centre, Hengbung, Senapati district, Manipur.E-mail: dr_anrao@rediffmail.com \\ ${ }^{2}$ Central National Herbarium, AJC Bose Indian Botanic Garden, Shibpur, Howrah-711 103, West Bengal, India. \\ E-mail:vmadhukar7@gmail.com
}

Keywords: Conservation, Manipur, new record, Orchidaceae, orchid checklist, threats.

Summary. Manipur is one of the eight states of North-East India placed inside square area $23^{\circ} 83^{\prime}-25^{\circ} 68^{\prime} \mathrm{N}$ and $93^{\circ} 03^{\prime}-94^{\circ} 78^{\prime} \mathrm{E}$. The natural vegetation of the state falls under the Indo-Myanmar Biodiversity Hotspot area with rich flora and fauna. In the present paper, 389 taxa of orchids (including 7 new records to Manipur) belonging to 90 genera, are listed and analysed. Various threats for the depletion of orchid species in the natural habitats of Manipur have been discussed and necessary conservation measures for their conservation are suggested.

\section{Дополненный список флоры орхидных Манипура}

\author{
А. Н. $\mathrm{Pao}^{1}$, В. Кумар ${ }^{2}$ \\ ${ }^{1}$ Центр по изучению и развитию орхидных, Хенгбунг, район Сенапати, Манипур \\ ${ }^{2}$ Центральныий национальный гербарий, Индийский ботанический сад, \\ Шибпур, Ховрах-711103, Западная Бенгалия, Индия
}

\begin{abstract}
Ключевые слова: лимитирующие факторы, Манипур, новая находка, сохранение, список орхидных, Orchidaceae.

Аннотация. Манипур - один из восьми штатов Северо-Восточной Индии, расположенный на площади между $23^{\circ} 83^{\prime}-25^{\circ} 68^{\prime}$ с.ш. и $93^{\circ} 03^{\prime}-94^{\circ} 78^{\prime}$ в.д. Естественная растительность штата попадает в область “IndoMyanmar Biodiversity Hotspot" с богатой флорой и фауной. В настоящей статье перечислены и проанализированы 389 таксонов орхидных (включая 7 новых находок для Манипура) из 90 родов. Обсуждаются различные лимитирующие факторы, приводящие к сокращению видов орхидных в естественных местообитаниях Манипура, и предлагаются необходимые меры по сохранению орхидных.
\end{abstract}

\section{Introduction}

Manipur is one of the eight states of North-East India placed at the eastern end of the region between the latitude of $23^{\circ} 83^{\prime}-25^{\circ} 68^{\prime} \mathrm{N}$ and longitude of $93^{\circ} 03^{\prime}-94^{\circ} 78^{\prime} \mathrm{E}$ and bordered by Nagaland on the north, Assam on the west and Myanmar on South and east (Fig. 1). The state is spreaded over an area of about 22347 sq. $\mathrm{km}$ of which about 14365 sq. $\mathrm{km}$ (nearly $64 \%$ of the total geographical area of the state) is covered by natural vegetation which may be classified into four types: "Tropical Semi-evergreen", "Dry Temperate", "Sub-Tropical Pine" and "Tropical Moist Deciduous". There are forests of teak, pine, oak, bamboo and cane. The natural vegetation of Manipur falls under the IndoMyanmar Biodiversity Hotspot area (Singh et al., 2010) with rich flora and fauna. 


\section{Materials and methods}

A critical review of literature (Hooker, 18881890; Pradhan, 1976, 1979; Shukla, Baishya, 1979; Ramakandtha, 1995; Phukan, 1996; Mao, 1999; Chauhan, 2001; Meitei et al., 2014; Laiphrakpam et al., 2014, 2015; Rao, Kumar, 2015; Sharma et al., 2015; Khuraijam, Roy, 2016) reveals that the state Manipur has been explored by several plant collectors and botanists since British period. Some of the important persons who made botanical collections from the state are George Watt in 1881-1882, C. B. Clarke in 1889, A. K. Meebold in 1906-1907, N. L. Bor in 1938-1945, K. Ward in 1952 (Kumar, Kumar, 2005) and subsequently during post independence period S. K. Mukerjee (1953), D. B. Deb (1956, 1957, 1961), Devi and Ghatak (1984), Ghatak and Devi (1986) and several botany scientists from Botanical Survey of India and other Research Institutions, Universities and

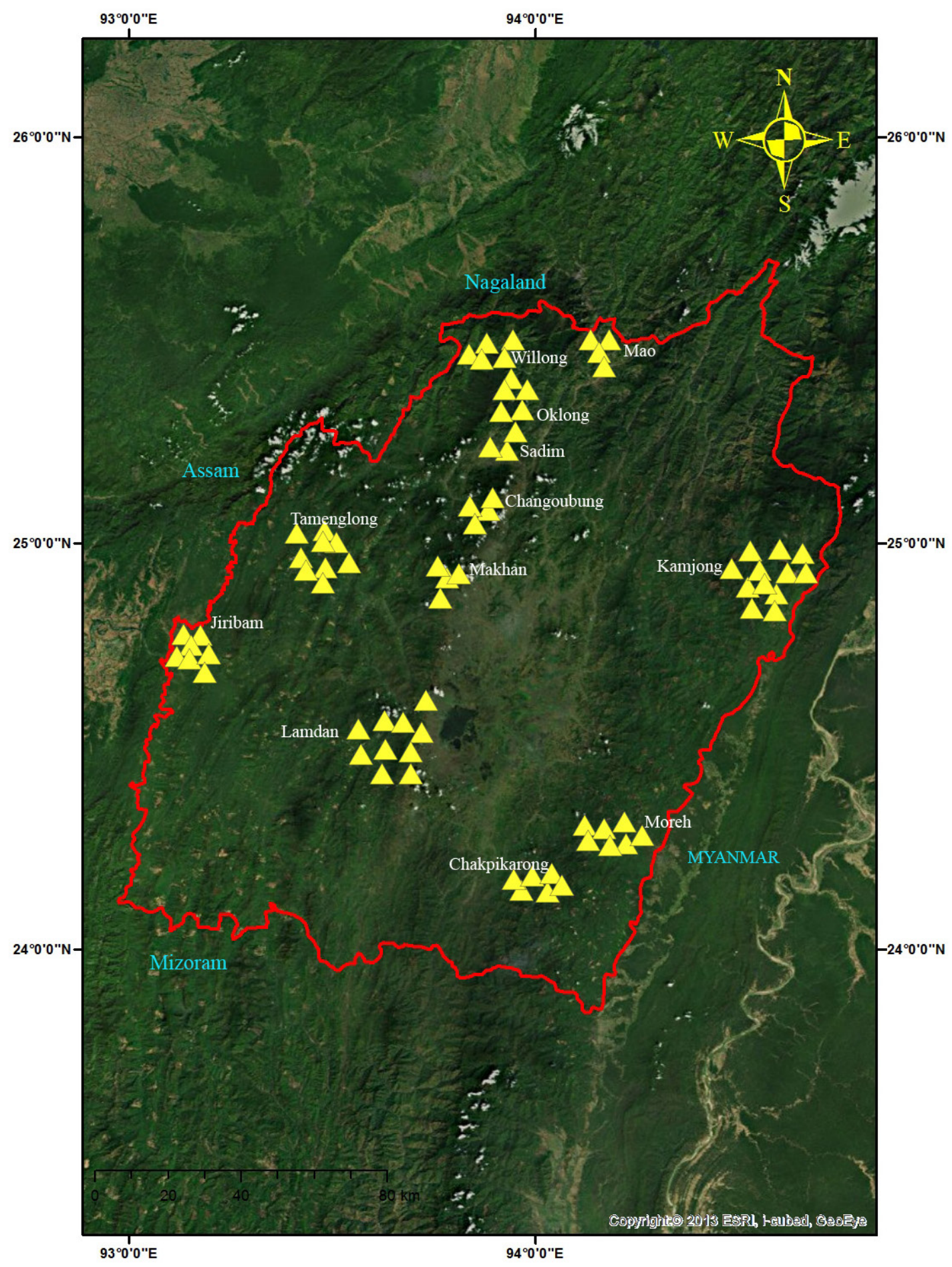

Fig. 1. Map of Manipur state showing the orchid survey areas in yellow triangle (Arc Map 10.5). 
plant collectors from various orchid nurseries of $\mathrm{N}$. E. Indian states.

During the last five years i. e. since 2012, a critical study has been conducted by the authors, based on the living collections which are made from different parts of Manipur and brought under cultivation at Orchid Research and Development Centre (ORDC), Hengbung, Manipur; herbarium collections deposited at Central National Herbarium (CAL), Howrah, West Bengal; BSI, Shillong (ASSAM), Meghalaya and COGCEHR Herbarium, Hengbung, Manipur; and consultation of many research papers
(Phukan, Mao, 2002a-b, 2004; Kumar, Kumar, 2005; Rao, 2007, 2015; Choltco, 2009; Chowdhery, 2009; Nanda et al., 2012a-b, 2013a-c, 2014a-d; Chowlu et al., 2012a-b, 2013a-e, 2014a-c, 2015a-c; Kishor et al., 2012, 2014; Yumkham et al., 2013; Chowlu, 2014, 2016; Chowlu, Rao, 2015a-b; Kumar, Rao, 2015; Kumar et al., 2015, 2016a-b, 2017; Rao et al., 2016; Kumar, 2017) on orchids of Manipur. The list of orchids reported so far from Manipur are listed in Table, nomenclature of each species is followed as per the World Checklist of Orchidaceae (Govaerts et al., 2018) and the analysis has been discussed below.

Table

List of Orchid species reported from Manipur

\begin{tabular}{|c|c|c|c|c|}
\hline $\begin{array}{l}\text { S. } \\
\text { No. }\end{array}$ & Species/variety & Habit & Voucher specimens record & $\begin{array}{l}\text { Reference of first } \\
\text { report for the state }\end{array}$ \\
\hline 1. & $\begin{array}{l}\text { Acampe carinata (Griff.) } \\
\text { Panigrahi }\end{array}$ & $\mathrm{E}$ & $\begin{array}{l}\text { Nanda et al. 00294, 00482, } \\
\text { 00051(all at COGCEHR) }\end{array}$ & Devi, Ghatak, 1984 \\
\hline 2. & Acampe ochracea (Lindl.) Hochr. & $\mathrm{E}$ & Nanda 00174 (COGCEHR) & Chauhan, 2001 \\
\hline 3. & $\begin{array}{l}\text { Acampe praemorsa (Roxb.) } \\
\text { Blatter et McCann var. rigida } \\
\text { (Smith) Barbhuiya et al. }\end{array}$ & $\mathrm{E}$ & $\begin{array}{l}\text { Herbarium not available. Live } \\
\text { plants under cultivation. }\end{array}$ & Devi, Ghatak, 1984 \\
\hline 4. & $\begin{array}{l}\text { Acanthophippium sylhetense } \\
\text { Lindl. }\end{array}$ & $\mathrm{T}$ & Herbarium not available. & Chauhan, 2001 \\
\hline 5. & Aerides multiflora Roxb. & $\mathrm{E}$ & Nanda et al. 40 (COGCEHR) & Devi, Ghatak, 1984 \\
\hline 6. & Aerides odorata Lour. & $\mathrm{E}$ & Nanda et al. 00323 (COGCEHR) & Devi, Ghatak, 1984 \\
\hline 7. & Aerides rosea Lindl. et Paxt. & $\mathrm{E}$ & Nanda et al. 00004 (COGCEHR) & Nanda et al., 2013c \\
\hline 8. & $\begin{array}{l}\text { Agrostophyllum callosum Rchb. } \\
\text { f. }\end{array}$ & $\mathrm{E}$ & $\begin{array}{l}\text { Kishor et al. 00258, } 00460 \text { (all at } \\
\text { COGCEHR). D. B. Deb 1528; A. } \\
\text { Meebold } 6904 \text { (all at CAL) }\end{array}$ & Mukerjee, 1953 \\
\hline 9. & $\begin{array}{l}\text { Agrostophyllum planicaulum J. } \\
\text { J. Sm. }\end{array}$ & $\mathrm{E}$ & Herbarium not available. & Clarke, 1889 \\
\hline 10. & Ania angustifolia Lindl. & $\mathrm{T}$ & $\begin{array}{l}\text { Nanda 00011; V. Kumar } 1658 \text { (all } \\
\text { at COGCEHR) }\end{array}$ & Nanda et al., 2013c \\
\hline 11. & $\begin{array}{l}\text { Aniaviridifusca (Hook.) Tang et } \\
\text { Summerh }\end{array}$ & $\mathrm{T}$ & George Watt $6293(\mathrm{~K})$ & Kumar, Kumar, 2005 \\
\hline 12. & $\begin{array}{l}\text { Anoectochilus roxburghii (Wall.) } \\
\text { Lindl. }\end{array}$ & $\mathrm{T}$ & Herbarium not available. & Clarke, 1889 \\
\hline 13. & Anthogonium gracile Lindl. & $\mathrm{T}$ & $\begin{array}{l}\text { V. Kumar 1656; Nanda } 00054 \text { (all } \\
\text { at COGCEHR) }\end{array}$ & Mukerjee, 1953 \\
\hline 14. & Aphyllorchis montana $\mathrm{Rchb} . \mathrm{f}$. & $\mathrm{M}$ & $\begin{array}{l}\text { V. Kumar 1660, } 1161 \\
\text { (COGCEHR) }\end{array}$ & Rao, Kumar, 2015 \\
\hline 15. & $\begin{array}{l}\text { Arachnis clarkei (Rchb. f.) J. J. } \\
\text { Sm. }\end{array}$ & $\mathrm{E}$ & Kishor et al. 00045 (COGCEHR) & Rao, 2007 \\
\hline 16. & $\begin{array}{l}\text { Arachnis labrosa (Lindl. et Paxt.) } \\
\text { Rchb. f. }\end{array}$ & $\mathrm{E}$ & C. Sathish Kumar 28747 (TBGT). & Kumar, Kumar, 2005 \\
\hline 17. & $\begin{array}{l}\text { Arachnis senapatianum (S. } \\
\text { Phukan et A. A. Mao) Kocyan et } \\
\text { Schuit. }\end{array}$ & $\mathrm{E}$ & A. A. Mao 60271 (CAL) & Phukan, Mao, 2002b \\
\hline 18. & $\begin{array}{l}\text { Arundina graminifolia (D. Don) } \\
\text { Hochr. }\end{array}$ & $\mathrm{T}$ & $\begin{array}{l}\text { Nanda et al. } 00157 \text { (COGCEHR). } \\
\text { D. B. Deb 306, 806, 1234, 2668; } \\
\text { S. K. Mukerjee 3121(all at CAL) }\end{array}$ & Mukerjee, 1953 \\
\hline
\end{tabular}


Continuation of table

\begin{tabular}{|c|c|c|c|c|}
\hline $\begin{array}{l}\text { S. } \\
\text { No. }\end{array}$ & Species/variety & Habit & Voucher specimens record & $\begin{array}{l}\text { Reference of first } \\
\text { report for the state }\end{array}$ \\
\hline 19. & $\begin{array}{l}\text { Brachycorythis galeandra (Rchb. } \\
\text { f.) Summerh. }\end{array}$ & $\mathrm{T}$ & S. K. Mukherjee 3118 (CAL) & Mukerjee, 1953 \\
\hline 20. & $\begin{array}{l}\text { Brachycorythis obcordata (Lindl.) } \\
\text { M. R. Almeida }\end{array}$ & $\mathrm{T}$ & Herbarium not available & $\begin{array}{l}\text { Laiphrakpam et al., } \\
2015\end{array}$ \\
\hline 21. & $\begin{array}{l}\text { Bryobium pudicum (Ridl.) Y. P. } \\
\text { Ng et Cribb }\end{array}$ & $\mathrm{E}$ & $\begin{array}{l}\text { Nanda et al. 0006, 506, } 000197 \\
\text { (all at COGCEHR) }\end{array}$ & Nanda et al., 2013c \\
\hline 22. & Bulbophyllum affine Lindl. & $\mathrm{E}$ & Chowlu 00021 (COGCEHR) & Chowlu et al., 2013e \\
\hline 23. & $\begin{array}{l}\text { Bulbophyllum appendiculatum } \\
\text { (Rolfe) J. J. Sm. }\end{array}$ & $\mathrm{E}$ & Kipgen 00569 (COGCEHR) & Chowlu et al., 2015c \\
\hline 24. & $\begin{array}{l}\text { Bulbophyllum bifurcatoflorens } \\
\text { (Fukuy.) J. J. Verm. et al. }\end{array}$ & $\mathrm{E}$ & $\begin{array}{l}\text { COGCEHR 00093; K. Chowlu } \\
\text { s. n. (acc. no. 399) [all at } \\
\text { COGCEHR] }\end{array}$ & Chowlu et al., 2012a \\
\hline 25. & $\begin{array}{l}\text { Bulbophyllum candidum (Lindl.) } \\
\text { Hook. f. }\end{array}$ & $\mathrm{E}$ & Kishor 00175 (COGCEHR) & Chowlu et al., 2013c \\
\hline 26. & $\begin{array}{l}\text { Bulbophyllum careyanum } \\
\text { (Hook.) Spreng. }\end{array}$ & $\mathrm{E}$ & $\begin{array}{l}\text { K. Chowlu 00050, 00138, } 00332 \\
\text { (all at COGCEHR) }\end{array}$ & Chauhan, 2001 \\
\hline 27. & $\begin{array}{l}\text { Bulbophyllum cariniflorum } \\
\text { Rchb. f. }\end{array}$ & $\mathrm{E}$ & K. Chowlu 00381 (COGCEHR) & Chowlu et al., 2013e \\
\hline 28. & $\begin{array}{l}\text { Bulbophyllum cornutum (Blume) } \\
\text { Rchb. f. }\end{array}$ & $\mathrm{E}$ & $\begin{array}{l}\text { Chowlu s. n. (acc. no. 394) } \\
\text { (COGCEHR) }\end{array}$ & $\begin{array}{l}\text { First time report in } \\
\text { present paper }\end{array}$ \\
\hline 29. & $\begin{array}{l}\text { Bulbophyllum cylindraceum } \\
\text { Lindl. }\end{array}$ & $\mathrm{E}$ & Chowlu 00411 (COGCEHR) & Chowlu, Rao, 2015a \\
\hline 30. & Bulbophyllum dickasonii Seidenf. & $\mathrm{E}$ & $\begin{array}{l}\text { Chowlu 00333(CAL). Chowlu } \\
000325 \text { (COGCEHR) }\end{array}$ & Chowlu et al., 2014a \\
\hline 31. & $\begin{array}{l}\text { Bulbophyllum dissitiflorum } \\
\text { Seidenf. }\end{array}$ & $\mathrm{E}$ & $\begin{array}{l}\text { Chowlu 00568, } 00571 \text { (all at } \\
\text { COGCEHR) }\end{array}$ & Chowlu et al., 2015c \\
\hline 32. & $\begin{array}{l}\text { Bulbophyllum elatum (Hook. f.) } \\
\text { J. J. Sm. }\end{array}$ & $\mathrm{E}$ & Herbarium not available. & Chauhan, 2001 \\
\hline 33. & Bulbophyllum forrestii Seidenf. & $\mathrm{E}$ & $\begin{array}{l}\text { Chowlu 00478, } 00536 \text { (all at } \\
\text { COGCEHR) }\end{array}$ & Chowlu, Rao, 2015a \\
\hline 34. & $\begin{array}{l}\text { Bulbophyllum gamblei (Hook. f.) } \\
\text { Hook. f. }\end{array}$ & $\mathrm{E}$ & $\begin{array}{l}\text { Kipgen 00547, 00548, } 00549 \text { (all } \\
\text { at COGCEHR) }\end{array}$ & Chowlu et al., 2015c \\
\hline 35. & $\begin{array}{l}\text { Bulbophyllum guttulatum (Hook. } \\
\text { f.) Balakr. }\end{array}$ & $\mathrm{E}$ & Shukla 63655 (ASSAM) & $\begin{array}{l}\text { Shukla, Baishya, } \\
1979\end{array}$ \\
\hline 36. & Bulbophyllum gymnopus Hook. f. & E & Herbarium not available. & Kumar, Kumar, 2005 \\
\hline 37. & $\begin{array}{l}\text { Bulbophyllum helenae (Kuntze) } \\
\text { J. J. Sm. }\end{array}$ & $\mathrm{E}$ & $\begin{array}{l}\text { Nanda 00386; Chowlu } 00387 \text { (all } \\
\text { at COGCEHR) }\end{array}$ & Chowlu, Rao, 2015a \\
\hline 38. & $\begin{array}{l}\text { Bulbophyllum hirtum }(\mathrm{Sm} .) \\
\text { Lindl. }\end{array}$ & $\mathrm{E}$ & Chowlu 00020 (COGCEHR) & Chowlu et al., 2013e \\
\hline 39. & Bulbophyllum khasyanum Griff. & $\mathrm{E}$ & Herbarium not available. & Kumar, Kumar, 2005 \\
\hline 40. & $\begin{array}{l}\text { Bulbophyllum kipgenii (Kishor et } \\
\text { al.) J. J. Verm. et al. }\end{array}$ & E & $\begin{array}{l}\text { COGCEHR/Herb/00001; Kishor } \\
00144 \text { (all at COGCEHR) }\end{array}$ & Kishor et al., 2012 \\
\hline 41. & $\begin{array}{l}\text { Bulbophyllum leopardinum } \\
\text { (Wall.) Lindl. }\end{array}$ & $\mathrm{E}$ & Herbarium not available. & Chauhan, 2001 \\
\hline 42. & Bulbophyllum lobbii Lindl. & $\mathrm{E}$ & Herbarium not available. & Chauhan, 2001 \\
\hline 43. & $\begin{array}{l}\text { Bulbophyllum moniliforme } \\
\text { Parish et Rchb. f. }\end{array}$ & $\mathrm{E}$ & Herbarium not available. & Chauhan, 2001 \\
\hline 44. & $\begin{array}{l}\text { Bulbophyllum odoratissimum } \\
\text { (Sm.) Lindl. }\end{array}$ & $\mathrm{E}$ & Chowlu 00221 (COGCEHR) & Chauhan, 2001 \\
\hline 45. & $\begin{array}{l}\text { Bulbophyllum penicillium C. S. } \\
\text { P. Parish et Rchb. f. }\end{array}$ & E & Chowlu 00361 (COGCEHR) & Chowlu, Rao, 2015a \\
\hline
\end{tabular}


Continuation of table

\begin{tabular}{|c|c|c|c|c|}
\hline $\begin{array}{l}\text { S. } \\
\text { No. }\end{array}$ & Species/variety & Habit & Voucher specimens record & $\begin{array}{l}\text { Reference of first } \\
\text { report for the state }\end{array}$ \\
\hline 46. & Bulbophyllum polyrrhizum Lindl. & $\mathrm{E}$ & Chowlu 00019 (COGCEHR) & Chowlu et al., 2013e \\
\hline 47. & $\begin{array}{l}\text { Bulbophyllum proboscideum } \\
\text { (Gagnep.) Seidenf. et Smitinand. }\end{array}$ & $\mathrm{E}$ & $\begin{array}{l}\text { Herbarium not available. Live } \\
\text { plants under cultivation. }\end{array}$ & Rao et al., 2016 \\
\hline 48. & Bulbophyllum propinquum Krzl. & $\mathrm{E}$ & Sathish Kumar 28755 (TBGT). & Kumar, Kumar, 2005 \\
\hline 49. & $\begin{array}{l}\text { Bulbophyllum reichenbachianum } \\
\text { Krzl. }\end{array}$ & $\mathrm{E}$ & $\begin{array}{l}\text { Under cultivation at Lyoyd } \\
\text { Botanic Garden, Darjeeling }\end{array}$ & Mukerjee, 1953 \\
\hline 50. & $\begin{array}{l}\text { Bulbophyllum reptans (Lindl.) } \\
\text { Lindl. }\end{array}$ & $\mathrm{E}$ & $\begin{array}{l}\text { K. Chowlu 00212, } 00213 \text { (all at } \\
\text { COGCEHR) }\end{array}$ & Chauhan, 2001 \\
\hline 51. & $\begin{array}{l}\text { Bulbophyllum retusiusculum } \\
\text { Rchb. f. }\end{array}$ & $\mathrm{E}$ & Chowlu et al. 00018 (COGCEHR) & Chowlu et al., 2013e \\
\hline 52. & $\begin{array}{l}\text { Bulbophyllum roseopictum J. J. } \\
\text { Verm., Schuit. et de Vogel }\end{array}$ & $\mathrm{E}$ & S. K. Mukerjee 3572 (CAL) & Mukerjee, 1953 \\
\hline 53. & $\begin{array}{l}\text { Bulbophyllum rothschildianum } \\
\text { (O'Brien) J. J. Sm. }\end{array}$ & E & $\begin{array}{l}\text { Chowlu 1651, } 00550 \text { (all at } \\
\text { COGCEHR) }\end{array}$ & Mao, 1999 \\
\hline 54. & Bulbophyllum rufinum Rchb. f. & $\mathrm{E}$ & Chowlu 00014 (COGCEHR) & Chowlu et al., 2013e \\
\hline 55. & Bulbophyllum scabratum Rchb. f. & $\mathrm{E}$ & $\begin{array}{l}\text { Chowlu 00145; Nanda s. n. (acc. } \\
\text { n. 360) [all at COGCEHR] }\end{array}$ & Chowlu et al., 2013e \\
\hline 56. & Bulbophyllum secundum Hook. f. & $\mathrm{E}$ & Chowlu 00107 (COGCEHR) & Chowlu et al., 2013e \\
\hline 57. & $\begin{array}{l}\text { Bulbophyllum sunipia J. J. Verm., } \\
\text { Schuit. et de Vogel }\end{array}$ & $\mathrm{E}$ & $\begin{array}{l}\text { B. Sharma 00349; K. Chowlu } 591 \\
\text { (all at COGCEHR) }\end{array}$ & Chowlu et al., 2013c \\
\hline 58. & Bulbophyllum triste Rchb. f. & $\mathrm{E}$ & Chowlu 00052 (COGCEHR) & Chauhan, 2001 \\
\hline 59. & Bulbophyllum umbellatum Lindl. & $\mathrm{E}$ & Chowlu 00124 (COGCEHR) & Chauhan, 2001 \\
\hline 60. & $\begin{array}{l}\text { Bulbophyllum xylophyllum C. S. } \\
\text { P. Parish et Rchb. f. }\end{array}$ & $\mathrm{E}$ & Chowlu 00016 (COGCEHR) & Chowlu et al., 2013e \\
\hline 61. & Calanthe biloba Lindl. & $\mathrm{T}$ & S. K. Mukerjee 3733 (CAL) & Mukerjee, 1953 \\
\hline 62. & Calanthe brevicornu Lindl. & $\mathrm{T}$ & D. B. Deb 2230, 1446 (CAL) & Mukerjee, 1953 \\
\hline 63. & Calanthe densiflora Lindl. & $\mathrm{T}$ & Herbarium not available. & Chauhan, 2001 \\
\hline 64. & Calanthe mannii Hook. f. & $\mathrm{T}$ & $\begin{array}{l}\text { Herbarium not available. Live } \\
\text { plants under cultivation. }\end{array}$ & Chauhan, 2001 \\
\hline 65. & Calanthe masuca (D. Don) Lindl. & $\mathrm{T}$ & Mao 106311(ASSAM) & Chauhan, 2001 \\
\hline 66. & Calanthe odora Griff. & $\mathrm{T}$ & G. Watt 6803 (CAL) & Mukerjee, 1953 \\
\hline 67. & Calanthe tricarinata Lindl. & $\mathrm{T}$ & Herbarium not available. & Chauhan, 2001 \\
\hline 68. & $\begin{array}{l}\text { Callostylis bambusifolia (Lindl.) } \\
\text { S. C. Chen et J. J. Wood }\end{array}$ & $\mathrm{E}$ & $\begin{array}{l}\text { Nanda 00072, } 00073 \text { (all at } \\
\text { COGCEHR) }\end{array}$ & Mao, 1999 \\
\hline 69. & Callostylis rigida Blume & $\mathrm{E}$ & Herbarium not available. & Kumar, Kumar, 2005 \\
\hline 70. & $\begin{array}{l}\text { Cephalanthera longifolia (L.) } \\
\text { Fritch }\end{array}$ & $\mathrm{T}$ & Herbarium not available. & Chauhan, 2001 \\
\hline 71. & $\begin{array}{l}\text { Cephalantheropsis longipes } \\
\text { (Hook. f.) Ormerod }\end{array}$ & $\mathrm{T}$ & Herbarium not available. & Deb, 1961 \\
\hline 72. & $\begin{array}{l}\text { Cephalantheropsis obcordata } \\
\text { (Lindl.) Ormerod }\end{array}$ & $\mathrm{T}$ & A. Mao 106408 (ASSAM) & Mukerjee, 1953 \\
\hline 73. & Ceratostylis himalaica Hook. f. & $\mathrm{E}$ & Chowlu s. n. (COGCEHR) & Chauhan, 2001 \\
\hline 74. & Chiloschistaparishii Seidenf. & $\mathrm{E}$ & Herbarium not available. & Chauhan, 2001 \\
\hline
\end{tabular}


Continuation of table

\begin{tabular}{|c|c|c|c|c|}
\hline $\begin{array}{l}\text { S. } \\
\text { No. }\end{array}$ & Species/variety & Habit & Voucher specimens record & $\begin{array}{l}\text { Reference of first } \\
\text { report for the state }\end{array}$ \\
\hline 75. & Chrysoglossum ornatum Blume & $\mathrm{T}$ & $\begin{array}{l}\text { Poornima, Bishwajit and Jennifer } \\
00437 \text { (COGCEHR) }\end{array}$ & Nanda et al., 2014c \\
\hline 76. & $\begin{array}{l}\text { Cleisocentron pallens (Lindl.) } \\
\text { Pears. et Cribb }\end{array}$ & $\mathrm{E}$ & Nanda et al. 00289 (COGCEHR) & $\begin{array}{l}\text { Laiphrakpam et al., } \\
2015\end{array}$ \\
\hline 77. & $\begin{array}{l}\text { Cleisomeria pilosulum (Gagnep.) } \\
\text { Seidenf. et Garay }\end{array}$ & $\mathrm{E}$ & $\begin{array}{l}\text { Thokchom } 00572 \text { (COGCEHR); } \\
\text { N.N. Rabha and L.R. Meitei } \\
131143 \text { (ASSAM) }\end{array}$ & Meitei et al., 2014 \\
\hline 78. & $\begin{array}{l}\text { Cleisostoma appendiculatum } \\
\text { (Lindl.) Jacks. }\end{array}$ & $\mathrm{E}$ & A. Mao 101944 (ASSAM) & Chauhan, 2001 \\
\hline 79. & Cleisostoma discolor Lindl. & E & Nanda et al. 00417 (COGCEHR) & Kumar, Kumar, 2005 \\
\hline 80. & $\begin{array}{l}\text { Cleisostoma filiforme (Lindl.) } \\
\text { Garay }\end{array}$ & $\mathrm{E}$ & Nanda et al. 596 (COGCEHR) & $\begin{array}{l}\text { Shukla, Baishya, } \\
1979\end{array}$ \\
\hline 81. & $\begin{array}{l}\text { Cleisostoma linearilobatum } \\
\text { (Seidenf. et Smit.) Garay }\end{array}$ & $\mathrm{E}$ & Herbarium not available. & $\begin{array}{l}\text { Live plants under } \\
\text { cultivation at ORDC } \\
\text { orchidarium }\end{array}$ \\
\hline 82. & $\begin{array}{l}\text { Cleisostoma racemiferum (Lindl.) } \\
\text { Garay }\end{array}$ & $\mathrm{E}$ & Kishor et al. 00300 (COGCEHR) & Chauhan, 2001 \\
\hline 83. & $\begin{array}{l}\text { Cleisostoma rolfeanum (King et } \\
\text { Pantl.) Garay }\end{array}$ & $\mathrm{E}$ & $\begin{array}{l}\text { C. Sathish Kumar } 28742 \text { (TBGT). } \\
\text { A. A. Mao 68282; A. K. Baisya } \\
72200 \text { (all at ASSAM) }\end{array}$ & Phukan, Mao, 2004 \\
\hline 84. & $\begin{array}{l}\text { Cleisostoma simondii (Gagnep.) } \\
\text { Seidenf. }\end{array}$ & $\mathrm{E}$ & $\begin{array}{l}\text { Herbarium not available. Live } \\
\text { plants under cultivation. }\end{array}$ & Rao, 2015 \\
\hline 85. & $\begin{array}{l}\text { Cleisostoma striatum (Rchb. f.) } \\
\text { N. E. Br. }\end{array}$ & $\mathrm{E}$ & Herbarium not available. & $\begin{array}{l}\text { Laiphrakpam et al., } \\
2015\end{array}$ \\
\hline 86. & $\begin{array}{l}\text { Cleisostomawilliamsonii (Rchb. } \\
\text { f.) Garay }\end{array}$ & $\mathrm{E}$ & $\begin{array}{l}\text { Nanda et al. s. n. (acc. no. 588) } \\
\text { [COGCEHR] }\end{array}$ & Kumar, Kumar, 2005 \\
\hline 87. & Coelogyne barbata Griff. & $\mathrm{E}$ & $\begin{array}{l}\text { George Watt 7452; Meebold 6926; } \\
\text { S. K. Mukerjee 3482; CAL) }\end{array}$ & Mukerjee, 1953 \\
\hline 88. & Coelogyne corymbosa Lindl. & E & $\begin{array}{l}\text { Chowlu et al. 18; Nanda s. n. (acc. } \\
\text { n. 11104) [all at COGCEHR]. S. } \\
\text { K. Mukerjee2812 (CAL) }\end{array}$ & Mukerjee, 1953 \\
\hline 89. & Coelogyne cristata Lindl. & $\mathrm{E}$ & $\begin{array}{l}\text { J. Devi } 2320 \text { (Manipur University } \\
\text { Herbarium) }\end{array}$ & Devi, Ghatak, 1984 \\
\hline 90. & Coelogyne fimbriata Lindl. & $\mathrm{E}$ & $\begin{array}{l}\text { Chowlu et al. s. n. (acc. no. 115) } \\
\text { [COGCEHR]. S. K. Mukerjee } \\
3721 \text { (CAL) }\end{array}$ & Mukerjee, 1953 \\
\hline 91. & Coelogyne flaccida Lindl. & $\mathrm{E}$ & $\begin{array}{l}\text { J. Devi } 2446 \text { (Manipur University } \\
\text { Herbarium) }\end{array}$ & Das, Jain, 1980 \\
\hline 92. & Coelogyne fuscescens Lindl. & $\mathrm{E}$ & $\begin{array}{l}\text { Chowlu et al. s. n. (acc. no. 440) } \\
\text { [COGCEHR] }\end{array}$ & Rao, 2015 \\
\hline 93. & $\begin{array}{l}\text { Coelogyne ghatakii T. K. Paul et } \\
\text { al. }\end{array}$ & $\mathrm{E}$ & $\begin{array}{l}\text { Ghatak 2213a (CAL). Ghatak } \\
\text { 2213b, c, d (Manipur Univ. } \\
\text { Herbarium) }\end{array}$ & $\begin{array}{l}\text { Paul et al., 1988; } \\
\text { Kumar, Kumar } 2005\end{array}$ \\
\hline 94. & Coelogyne griffithii Hook. f. & $\mathrm{E}$ & Watt's collection $6780(\mathrm{CAL})$ & Hooker, 1890 \\
\hline 95. & $\begin{array}{l}\text { Coelogyne holochila Hunt et } \\
\text { Summerh. }\end{array}$ & $\mathrm{E}$ & Herbarium not available. & Kumar, Kumar, 2005 \\
\hline 96. & Coelogyne longipes Lindl. & $\mathrm{E}$ & $\begin{array}{l}\text { S. K. Mukerjee 3439; S. C. Sinha } \\
1893 \text { (all at CAL) }\end{array}$ & Mukerjee, 1953 \\
\hline 97. & Coelogyne micrantha Lindl. & $\mathrm{E}$ & $\begin{array}{l}\text { Chowlu et al. 00329, } 00141 \text { (all at } \\
\text { COGCEHR) }\end{array}$ & Das, Jain, 1980 \\
\hline 98. & Coelogyne nitida (D. Don) Lindl. & E & $\begin{array}{l}\text { Nanda et al. 00028; Chowlu } 00032 \\
\text { (all at COGCEHR) }\end{array}$ & Devi, Ghatak, 1984 \\
\hline
\end{tabular}




\section{Continuation of table}

\begin{tabular}{|c|c|c|c|c|}
\hline $\begin{array}{c}\text { S. } \\
\text { No. }\end{array}$ & Species/variety & Habit & Voucher specimens record & $\begin{array}{l}\text { Reference of first } \\
\text { report for the state }\end{array}$ \\
\hline 99. & Coelogyne ovalis Lindl. & $\mathrm{E}$ & $\begin{array}{l}\text { Chowlu et al. s. n. (acc. no. 97); K. } \\
\text { Chowlu } 00180 \text { [all at COGCEHR] }\end{array}$ & Mukerjee, 1953 \\
\hline 100. & $\begin{array}{l}\text { Coelogyne ovalis Lindl. var. } \\
\text { latifolia Hook. f. }\end{array}$ & $\mathrm{E}$ & Herbarium not available. & Hooker, 1890 \\
\hline 101. & Coelogyne prolifera Lindl. & $\mathrm{E}$ & $\begin{array}{l}\text { Nanda et al. 00025, 00033; } \\
\text { Chowlu 00026; Kishor et al. } \\
00027 \text { (all at COGCEHR). A. } \\
\text { Meebold } 4766 \text { (CAL) }\end{array}$ & Devi, Ghatak, 1984 \\
\hline 102. & $\begin{array}{l}\text { Coelogyne pulverula Teijm. et } \\
\text { Binnend. }\end{array}$ & $\mathrm{E}$ & Herbarium not available & Ghatak, Devi, 1986 \\
\hline 103. & Coelogyne punctulata Lindl. & $\mathrm{E}$ & $\begin{array}{l}\text { Chowlu 00030, } 00038 \text { (all at } \\
\text { COGCEHR) }\end{array}$ & Das, Jain, 1980 \\
\hline 104. & Coelogyne schultesii Jain et Das & $\mathrm{E}$ & Meebold $4766(\mathrm{CAL})$ & Das, Jain, 1980 \\
\hline 105. & Coelogyne stricta (D. Don) Schltr. & $\mathrm{E}$ & $\begin{array}{l}\text { Imbeng and Atan s. n. (acc. no. } \\
\text { 226) [COGCEHR] }\end{array}$ & Das, Jain, 1980 \\
\hline 106. & $\begin{array}{l}\text { Coelogyne suaveolens (Lindl.) } \\
\text { Hook. f. }\end{array}$ & $\mathrm{E}$ & $\begin{array}{l}\text { Nanda et al. 00035; Chowlu et al. } \\
1196 \text { (all at COGCEHR) }\end{array}$ & $\begin{array}{l}\text { Shukla, Baishya, } \\
1979\end{array}$ \\
\hline 107. & Coelogyne trinervis Lindl. & $\mathrm{E}$ & Herbarium not available. & Chauhan, 2001 \\
\hline 108. & Coelogyne viscosa Rchb. $\mathrm{f}$. & $\mathrm{E}$ & $\begin{array}{l}\text { Kishor et al. 00022; Chowlu } \\
00173,00023 \text { (all at COGCEHR) }\end{array}$ & $\begin{array}{l}\text { Shukla, Baishya, } \\
1979\end{array}$ \\
\hline 109. & $\begin{array}{l}\text { Conchidium muscicola (Lindl.) } \\
\text { Rauscht. }\end{array}$ & $\mathrm{E}$ & D. B. Deb 935 CAL) & Deb, 1961 \\
\hline 110. & $\begin{array}{l}\text { Corymborkis veratrifolia (Reinw.) } \\
\text { Blume }\end{array}$ & $\mathrm{T}$ & Herbarium not available & Chauhan, 2001 \\
\hline 111. & $\begin{array}{l}\text { Crepidium acuminatum (D. Don) } \\
\text { Szlach. }\end{array}$ & $\mathrm{T}$ & $\begin{array}{l}\text { V. Kumar 1665, } 1666 \text { (all at } \\
\text { COGCEHR); }\end{array}$ & Mukerjee, 1953 \\
\hline 112. & $\begin{array}{l}\text { Crepidium calophyllum (Rchb. f.) } \\
\text { Szlach. }\end{array}$ & $\mathrm{T}$ & $\begin{array}{l}\text { Herbarium not available. Live } \\
\text { plants under cultivation. }\end{array}$ & Rao, 2015 \\
\hline 113. & $\begin{array}{l}\text { Crepidium josephianum (Rchb. } \\
\text { f.) Marg. }\end{array}$ & $\mathrm{T}$ & Not seen by authors. & Chauhan, 2001 \\
\hline 114. & $\begin{array}{l}\text { Crepidium khasianum (Hook. f.) } \\
\text { Szlach. }\end{array}$ & $\mathrm{T}$ & $\begin{array}{l}\text { Herbarium not available. Live } \\
\text { plants under cultivation. }\end{array}$ & Rao, 2015 \\
\hline 115. & $\begin{array}{l}\text { Crepidium purpureum (Lindl.) } \\
\text { Szlach. }\end{array}$ & $\mathrm{T}$ & $\begin{array}{l}\text { K. Chowlu s. n. (acc. no. 365) } \\
\text { [COGCEHR] }\end{array}$ & Rao, 2015 \\
\hline 116. & Cryptochilus lutea Lindl. & $\mathrm{E}$ & Herbarium not available. & Chauhan, 2001 \\
\hline 117. & Cryptochilus sanguinea Lindl. & $\mathrm{E}$ & S. K. Mukerjee 3042 (CAL) & Mukerjee, 1953 \\
\hline 118. & Cymbidium aloifolium (L.) Sw. & $\mathrm{E}$ & Chowlu 00012 (COGCEHR) & Devi, Ghatak, 1984 \\
\hline 119. & Cymbidium cochleare Lindl. & $\mathrm{E}$ & Chowlu-00439 (COGCEHR) & Chowlu et al., 2013d \\
\hline 120. & $\begin{array}{l}\text { Cymbidium concinnum Liu et } \\
\text { Chen }\end{array}$ & $\mathrm{E}$ & Chowlu 1562 (COGCEHR) & Chowlu et al., 2015b \\
\hline 121. & Cymbidium crassifolium Herb. & $\mathrm{E}$ & $\begin{array}{l}\text { Nanda et al. 00036; Chowlu } 00170 \\
\text { (all at COGCEHR) }\end{array}$ & Kumar, Kumar, 2005 \\
\hline 122. & Cymbidium cyperifolium Lindl. & $\mathrm{T}$ & Herbarium not available. & Devi, Ghatak, 1984 \\
\hline 123. & Cymbidium dayanum Rchb. f. & $\mathrm{E}$ & $\begin{array}{l}\text { OBDD-IBSD-K-C-01 to OBDD- } \\
\text { IBSD-K-C-06 }\end{array}$ & Kumar et al., 2018 \\
\hline 124. & Cymbidium devonianum Paxt. & $\mathrm{E}$ & $\begin{array}{l}\text { Herbarium not available. Live } \\
\text { plants under cultivation. }\end{array}$ & Chauhan, 2001 \\
\hline
\end{tabular}


Continuation of table

\begin{tabular}{|c|c|c|c|c|}
\hline $\begin{array}{l}\text { S. } \\
\text { No. }\end{array}$ & Species/variety & Habit & Voucher specimens record & $\begin{array}{l}\text { Reference of first } \\
\text { report for the state }\end{array}$ \\
\hline 125. & Cymbidium eburneum Lindl. & $\mathrm{E}$ & $\begin{array}{l}\text { J. Devi } 2389 \text { (Manipur University } \\
\text { Herbarium) }\end{array}$ & Devi, Ghatak, 1984 \\
\hline 126. & Cymbidium elegans Lindl. & E & A. Mao 106309 (ASSAM) & $\begin{array}{l}\text { Mukerjee, 1953; A. } \\
\text { A. Mao, } 1999\end{array}$ \\
\hline 127. & Cymbidium ensifolium $\mathrm{Sw}$. & $\mathrm{T}$ & Chowlu 971 (COGCEHR) & Ghatak, Devi, 1986 \\
\hline 128. & Cymbidium erythraeum Lindl. & $\mathrm{E}$ & $\begin{array}{l}\text { Herbarium not available. Live } \\
\text { plants under cultivation. }\end{array}$ & Rao, 2007 \\
\hline 129. & $\begin{array}{l}\text { Cymbidium hengbungense } \mathrm{A} . \mathrm{N} \text {. } \\
\text { Rao et al. }\end{array}$ & $M$ & A. N. Rao 00409 (COGCEHR) & Rao et al., 2016 \\
\hline 130. & $\begin{array}{l}\text { Cymbidium hookerianum Rchb. } \\
\text { f. }\end{array}$ & $\mathrm{E}$ & $\begin{array}{l}\text { S. K. Mukherjee 3068, } 3640 \text { (all } \\
\text { at CAL) }\end{array}$ & Mukerjee, 1953 \\
\hline 131. & Cymbidium insigne Rolfe & $\mathrm{E}$ & Herbarium not available. & Kumar, Kumar, 2005 \\
\hline 132. & Cymbidium irdioides D. Don & $\mathrm{E}$ & Chowlu 00435 (COGCEHR) & Devi, Ghatak, 1984 \\
\hline 133. & Cymbidium lancifolium Hook. f. & $\mathrm{T}$ & Nanda et al. 00029 (COGCEHR) & Chauhan, 2001 \\
\hline 134. & Cymbidium macrorhizon Lindl. & M & Herbarium not available & Rao, 2007 \\
\hline 135. & Cymbidium mastersii Lindl. & $\mathrm{E}$ & Herbarium not available. & Pradhan, 1979 \\
\hline 136. & $\begin{array}{l}\text { Cymbidium munronianum Kingh } \\
\text { et Pantl. }\end{array}$ & $\mathrm{T}$ & Herbarium not available. & Ghatak, Devi, 1986 \\
\hline 137. & $\begin{array}{l}\text { Cymbidium nanulum } \mathrm{Wu} \text { et S. C. } \\
\text { Chen }\end{array}$ & $\mathrm{T}$ & V. Kumar 74200 (CAL) & Kumar, 2017 \\
\hline 138. & $\begin{array}{l}\text { Cymbidium sinense (Jacks.) } \\
\text { Willd. }\end{array}$ & $\mathrm{T}$ & $\begin{array}{l}\text { Hengbung (under cultivation at } \\
\text { ORDC Orchidarium). }\end{array}$ & $\begin{array}{l}\text { Chowlu et al., 2014; } \\
\text { Rao, } 2015\end{array}$ \\
\hline 139. & Cymbidium tigrinum Hook. f. & $\mathrm{L}$ & Kishor 00223 (COGCEHR) & Kumar, Kumar, 2005 \\
\hline 140. & Cyrtosia nana (Downie) Garay & M & $\begin{array}{l}\text { P. K. Singh } 000816 \text { (Man. Univ. } \\
\text { Mus. Plants) }\end{array}$ & $\begin{array}{l}\text { Sanatombi et al., } \\
2013\end{array}$ \\
\hline 141. & $\begin{array}{l}\text { Dendrobium abhaycharanii } \\
\text { (Phukan et A. A. Mao) Schuit. et } \\
\text { P. B. Adams }\end{array}$ & $\mathrm{E}$ & $\begin{array}{l}\text { V. Kumar 1681 (COGCEHR); } \\
\text { Nanda et al. s. n. (acc. no. 578) [all } \\
\text { at COGCEHR] }\end{array}$ & Phukan, Mao, 2005 \\
\hline 142. & Dendrobium aduncum Lindl. & $\mathrm{E}$ & $\begin{array}{l}\text { Herbarium not available. Live } \\
\text { plants under cultivation. }\end{array}$ & $\begin{array}{l}\text { Laiphrakpam et al., } \\
2015\end{array}$ \\
\hline 143. & $\begin{array}{l}\text { Dendrobium albopurpureum } \\
\text { (Seidenf.) Schuit. et P. B. Adams }\end{array}$ & $\mathrm{E}$ & $\begin{array}{l}\text { Y. Nanda, Krishna, Bishwajit } \\
\text { and Jennifer 00438; Kishor et al. } \\
00066 \text { (all at COGCEHR) }\end{array}$ & Nanda et al., 2014c \\
\hline 144. & Dendrobium amoenum Lindl. & $\mathrm{E}$ & Y. Nanda 00372 (COGCEHR) & Nanda et al., 2013b \\
\hline 145. & Dendrobium amplum Lindl. & $\mathrm{E}$ & Chowlu 00102 (COGCEHR) & Mao, 1999 \\
\hline 146. & Dendrobium anceps Sw. & $\mathrm{E}$ & Herbarium not available & Kumar, Kumar, 2005 \\
\hline 147. & $\begin{array}{l}\text { Dendrobium aphyllum (Roxb.) } \\
\text { Fischer }\end{array}$ & $\mathrm{E}$ & $\begin{array}{l}\text { Herbarium not available. Live } \\
\text { plants under cultivation. }\end{array}$ & Devi, Ghatak, 1984 \\
\hline 148. & Dendrobium bellatulum Rolfe & $\mathrm{E}$ & Kishor et al. 144 (COGCEHR) & Pradhan, 1979 \\
\hline 149. & Dendrobium bensonae Rchb. f. & $\mathrm{E}$ & $\begin{array}{l}\text { Herbarium not available. Live } \\
\text { plants under cultivation. }\end{array}$ & Pradhan, 1979 \\
\hline 150. & Dendrobium bicameratum Lindl. & $\mathrm{E}$ & $\begin{array}{l}\text { Nanda 00024; Kishor } 00263 \text { (all at } \\
\text { COGCEHR) }\end{array}$ & Nanda et al., 2012b \\
\hline 151. & $\begin{array}{l}\text { Dendrobium brymerianum } \text { Rchb. } \\
\text { f. }\end{array}$ & $\mathrm{E}$ & $\begin{array}{l}\text { Kishor et al. 00434, 00463, } 00464 \\
\text { (all at COGCEHR) }\end{array}$ & Mao, 1999 \\
\hline
\end{tabular}


Continuation of table

\begin{tabular}{|c|c|c|c|c|}
\hline $\begin{array}{l}\text { S. } \\
\text { No. }\end{array}$ & Species/variety & Habit & Voucher specimens record & $\begin{array}{l}\text { Reference of first } \\
\text { report for the state }\end{array}$ \\
\hline 152. & Dendrobium capillipes Rchb. f. & $\mathrm{E}$ & $\begin{array}{l}\text { Y. Nanda 00371, } 00032 \text { (all at } \\
\text { COGCEHR) }\end{array}$ & Nanda et al., $2013 \mathrm{~b}$ \\
\hline 153. & Dendrobium cariniferum Rchb. f. & $\mathrm{E}$ & G. Watt 6500 (CAL) & Mukerjee, 1953 \\
\hline 154. & Dendrobium chapaense Aver. & $\mathrm{E}$ & $\begin{array}{l}\text { COGCEHR/Herb/00003 (ASSAM, } \\
\text { CAL) }\end{array}$ & Kishor et al., 2013 \\
\hline 155. & Dendrobium chrysanthum Lindl. & $\mathrm{E}$ & $\begin{array}{l}\text { Nanda } 000483 \text { (COGCEHR); } \\
\text { Nanda et al. } 00062 \text { (COGCEHR) }\end{array}$ & Mao, 1999 \\
\hline 156. & Dendrobium chryseum Rolfe & $\mathrm{E}$ & $\begin{array}{l}\text { Herbarium not available. Live } \\
\text { plants under cultivation. }\end{array}$ & Deori, 2007 \\
\hline 157. & Dendrobium chrysotoxum Lindl. & $\mathrm{E}$ & Kishor et al. 324 (COGCEHR) & Mukerjee, 1953 \\
\hline 158. & $\begin{array}{l}\text { Dendrobium crepidatum Lindl. } \\
\text { et Paxt. }\end{array}$ & $\mathrm{E}$ & $\begin{array}{l}\text { Herbarium not available. Live } \\
\text { plants under cultivation. }\end{array}$ & Devi, Ghatak, 1984 \\
\hline 159. & $\begin{array}{l}\text { Dendrobium crystallinum Rchb. } \\
\text { f. }\end{array}$ & $\mathrm{E}$ & Nanda 00370 (COGCEHR) & Nanda et al., 2013b \\
\hline 160. & Dendrobium cumulatum Lindl. & $\mathrm{E}$ & Nanda et al. 1151 (COGCEHR) & Nanda et al., 2014c \\
\hline 161. & Dendrobium dantaniense Guill. & $\mathrm{E}$ & Deori 101148 (ASSAM) & Deori, 2007 \\
\hline 162. & Dendrobium delacourii Guill. & $\mathrm{E}$ & $\begin{array}{l}\text { N. N. Rabha and L. R. Meitei } \\
131115 \text { (ASSAM) }\end{array}$ & Meitei et al., 2014 \\
\hline 163. & Dendrobium densiflorum Lindl. & $\mathrm{E}$ & $\begin{array}{l}\text { Kishor et al. s. n. (acc. no. } \\
\text { 311); Nanda et al. } 00068 \text { (all at } \\
\text { COGCEHR) }\end{array}$ & Mao, 1999 \\
\hline 164. & Dendrobium denudans D. Don & $\mathrm{E}$ & $\begin{array}{l}\text { Nanda et al. 00065; Kishor et al. } \\
317,321 \text { (all at COGCEHR) }\end{array}$ & Mukerjee, 1953 \\
\hline 165. & Dendrobium devonianum Paxt. & $\mathrm{E}$ & Nanda et al. 00050 (COGCEHR) & Devi, Ghatak, 1984 \\
\hline 166. & $\begin{array}{l}\text { Dendrobium dickasonii L. O. } \\
\text { Williams. }\end{array}$ & $\mathrm{E}$ & Pradhan 6 (K-sprit collection) & Pradhan, 1976 \\
\hline 167. & Dendrobium draconis Rchb. $\mathrm{f}$. & $\mathrm{E}$ & $\begin{array}{l}\text { J. Devi } 2451 \text { (Manipur Univ. } \\
\text { Herbarium) }\end{array}$ & Pradhan, 1976 \\
\hline 168. & Dendrobium eriiflorum Griff. & $\mathrm{E}$ & Nanda 00060 (COGCEHR) & Mukerjee, 1953 \\
\hline 169. & Dendrobium falconeri Hook. & $\mathrm{E}$ & $\begin{array}{l}\text { Nanda et al. 00061; Kishor et al. } \\
301 \text { (all at COGCEHR) }\end{array}$ & Hooker, 1890 \\
\hline 170. & Dendrobium farmerii Paxt. & $\mathrm{E}$ & $\begin{array}{l}\text { J. Devi } 2303 \text { (Manipur Univ. } \\
\text { Herbarium) }\end{array}$ & Devi, Ghatak, 1984 \\
\hline 171. & Dendrobium fimbriatum Hook. & $\mathrm{E}$ & $\begin{array}{l}\text { Y. Nanda 00310, } 00284 \text { (at } \\
\text { COGCEHR) }\end{array}$ & Deori, 2007 \\
\hline 172. & $\begin{array}{l}\text { Dendrobium fimbriatum Hook. } \\
\text { var. oculatum Hook. }\end{array}$ & $\mathrm{E}$ & $\begin{array}{l}\text { J. Devi } 2461 \text { (Manipur Univ. } \\
\text { Herbarium) }\end{array}$ & Devi, Ghatak, 1984 \\
\hline 173. & Dendrobium formosum Lindl. & $\mathrm{E}$ & Supt. G. Gardner s. n. (CAL) & Devi, Ghatak, 1984 \\
\hline 174. & Dendrobium fugax Rchb. f. & $\mathrm{E}$ & $\begin{array}{l}\text { Herbarium not available. Live } \\
\text { plants under cultivation. }\end{array}$ & Chauhan, 2001 \\
\hline 175. & Dendrobium fuscescens Griff. & $\mathrm{E}$ & $\begin{array}{l}\text { Herbarium not available. Live } \\
\text { plants under cultivation. }\end{array}$ & Rao, 2007 \\
\hline 176. & Dendrobium gibsonii Lindl. & $\mathrm{E}$ & Nanda et al. 904 (COGCEHR) & Devi, Ghatak, 1984 \\
\hline 177. & $\begin{array}{l}\text { Dendrobium gratiotissimum } \\
\text { Rchb. f. }\end{array}$ & $\mathrm{E}$ & $\begin{array}{l}\text { J. Devi } 2362 \text { Minipur Univ. } \\
\text { Herbarium) }\end{array}$ & Pradhan, 1979 \\
\hline 178. & $\begin{array}{l}\text { Dendrobium hesperis (Seidenf.) } \\
\text { Schuit. et P. B. Adams }\end{array}$ & $\mathrm{E}$ & Nanda et al. 00480 (COGCEHR) & Nanda et al., 2014b \\
\hline 179. & Dendrobium heterocarpum Lindl. & $\mathrm{E}$ & Nanda et al. 39 (COGCEHR) & Devi, Ghatak, 1984 \\
\hline
\end{tabular}


Continuation of table

\begin{tabular}{|c|c|c|c|c|}
\hline $\begin{array}{c}\text { S. } \\
\text { No. }\end{array}$ & Species/variety & Habit & Voucher specimens record & $\begin{array}{l}\text { Reference of first } \\
\text { report for the state }\end{array}$ \\
\hline 180. & Dendrobium hookerianum Lindl. & $\mathrm{E}$ & $\begin{array}{l}\text { Herbarium not available. Live } \\
\text { plants under cultivation. }\end{array}$ & Chauhan, 2001 \\
\hline 181. & Dendrobium infundibulum Lindl. & $\mathrm{E}$ & C. Sathish Kumar 28726 (TBGT). & Pradhan, 1979 \\
\hline 182. & Dendrobium jenkinsii Lindl. & $\mathrm{E}$ & Nanda 00415 (COGCEHR) & Chauhan, 2001 \\
\hline 183. & Dendrobium lindleyii Steud. & $\mathrm{E}$ & Nanda et al. s. n. (COGCEHR) & Devi, Ghatak, 1984 \\
\hline 184. & Dendrobium linguella Rchb. f. & $\mathrm{E}$ & Herbarium not available & Ghatak, Devi, 1986 \\
\hline 185. & Dendrobium lituiflorum Lindl. & $\mathrm{E}$ & $\begin{array}{l}\text { Kishor et al. s. n. (acc. no. 574) } \\
\text { [COGCEHR] }\end{array}$ & Mukerjee, 1953 \\
\hline 186. & Dendrobium longicornu Lindl. & $\mathrm{E}$ & $\begin{array}{l}\text { Y. Nanda 00414; Kishor et al. } \\
00273,00315 \text { (all at COGCEHR) }\end{array}$ & Mukerjee, 1953 \\
\hline 187. & Dendrobium mannii Ridl. & $\mathrm{E}$ & J. S. Khuraijam 101968 (LWG) & $\begin{array}{l}\text { Khuraijam, Roy, } \\
2016\end{array}$ \\
\hline 188. & $\begin{array}{l}\text { Dendrobium moniliforme (L.) } \\
\text { Sw. }\end{array}$ & $\mathrm{E}$ & S. K. Mukerjee 2855 (CAL) & Mukerjee, 1953 \\
\hline 189. & $\begin{array}{l}\text { Dendrobium moschatum (Buch.- } \\
\text { Ham.) Sw. }\end{array}$ & $\mathrm{E}$ & S. K. Mukerjee 2849 (CAL) & Mukerjee, 1953 \\
\hline 190. & Dendrobium nobile Lindl. & $\mathrm{E}$ & Nanda et al. s. n. (COGCEHR) & Mukerjee, 1953 \\
\hline 191. & Dendrobium ochreatum Lindl. & $\mathrm{E}$ & Nanda et al. 00182 (COGCEHR) & Mukerjee, 1953 \\
\hline 192. & Dendrobium parcum Rchb. f. & $\mathrm{E}$ & $\begin{array}{l}\text { Nanda et al. 00275, 00064, } 00481 \\
\text { (all at COGCEHR) }\end{array}$ & Kumar, Kumar, 2005 \\
\hline 193. & Dendrobium parishii Rchb. f. & $\mathrm{E}$ & $\begin{array}{l}\text { J. Devi } 2369 \text { (Manipur Univ. } \\
\text { Herbarium) }\end{array}$ & Devi, Ghatak, 1984 \\
\hline 194. & Dendrobium pendulum Roxb. & $\mathrm{E}$ & Herbarium not available & Pradhan, 1979 \\
\hline 195. & Dendrobium polyanthum Lindl. & $\mathrm{E}$ & $\begin{array}{l}\text { Nanda et al. s. n. (acc. no. 536) } \\
\text { [COGCEHR] }\end{array}$ & Devi, Ghatak,1984 \\
\hline 196. & $\begin{array}{l}\text { Dendrobium porphyrochilum } \\
\text { Lindl. }\end{array}$ & $\mathrm{E}$ & V. Kumar 1662 (COGCEHR) & Mukerjee, 1953 \\
\hline 197. & Dendrobium pulchellum Lindl. & $\mathrm{E}$ & Nanda et al. s. n. (COGCEHR) & Mukerjee, 1953 \\
\hline 198. & $\begin{array}{l}\text { Dendrobium salaccense (Blume) } \\
\text { Lindl. }\end{array}$ & $E$ & Y. Nanda 197 (COGCEHR) & Nanda et al., 2013b \\
\hline 199. & $\begin{array}{l}\text { Dendrobium sinominutiflorum S. } \\
\text { C. Chen et al. }\end{array}$ & E & $\begin{array}{l}\text { Nanda 00405, Akhimpau s. n. (acc. } \\
\text { no. 584) [all at COGCEHR] }\end{array}$ & $\begin{array}{l}\text { Nanda et al., 2014; } \\
\text { Rao et al. } 2016\end{array}$ \\
\hline 200 . & Dendrobium spatella Rchb. f. & E & $\begin{array}{l}\text { Nanda et al. } 00059,1065 \text { (all at } \\
\text { COGCEHR). }\end{array}$ & Chauhan, 2001 \\
\hline 201. & Dendrobium stuposum Lindl. & $\mathrm{E}$ & Kishor et al. 00259 (COGCEHR) & Chauhan, 2001 \\
\hline 202. & Dendrobium sulcatum Lindl. & $\mathrm{E}$ & A. Mao 101934 (ASSAM) & Chauhan, 2001 \\
\hline 203. & $\begin{array}{l}\text { Dendrobium thyrsiflorum B. S. } \\
\text { Williams }\end{array}$ & $\mathrm{E}$ & $\begin{array}{l}\text { J. Devi } 2370 \text { Manipur University } \\
\text { Herbarium) }\end{array}$ & Mukerjee, 1953 \\
\hline 204. & Dendrobium transparens Lindl. & $\mathrm{E}$ & Nanda et al. 00453 (COGCEHR) & Mao, 1999 \\
\hline 205. & Dendrobium wardianum Warner & $\mathrm{E}$ & Kishor 00048 (COGCEHR) & Pradhan, 1979 \\
\hline 206. & $\begin{array}{l}\text { Dendrobium wattii (Hook. f.) } \\
\text { Rchb. f. }\end{array}$ & $\mathrm{E}$ & G. Watt 5944 (CAL) & Hooker, 1890 \\
\hline 207. & $\begin{array}{l}\text { Dendrobium williamsonii Day et } \\
\text { Rchb. f. }\end{array}$ & E & $\begin{array}{l}\text { Kishor et al. 508, } 509 \text { (all at } \\
\text { COGCEHR) }\end{array}$ & $\begin{array}{l}\text { Shukla, Baishya, } \\
1979\end{array}$ \\
\hline 208. & $\begin{array}{l}\text { Dickasonia vernicosa } \text { L. O. } \\
\text { Williams }\end{array}$ & $E$ & Herbarium not available & Pradhan, 1979 \\
\hline
\end{tabular}


Continuation of table

\begin{tabular}{|c|c|c|c|c|}
\hline $\begin{array}{c}\text { S. } \\
\text { No. }\end{array}$ & Species/variety & Habit & Voucher specimens record & $\begin{array}{l}\text { Reference of first } \\
\text { report for the state }\end{array}$ \\
\hline 209. & Dienia ophrydis (Koen.) Seidenf. & $\mathrm{T}$ & Chowlu s. n. (acc. no. 479) & Mukerjee, 1953 \\
\hline 210. & Eria biflora Griff. & $\mathrm{E}$ & Nanda et al. 00005 (COGCEHR) & Nanda et al., 2013c \\
\hline 211. & Eria clavicaulis Lindl. & $\mathrm{E}$ & $\begin{array}{l}\text { Nanda 00333, 00365, } 00395 \text { (all at } \\
\text { COGCEHR) }\end{array}$ & Nanda et al., 2013a \\
\hline 212. & Eria coronaria (Lindl.) Rchb. f. & $\mathrm{E}$ & $\begin{array}{l}\text { Nanda et al. 00068; Kishor et al. } \\
00278 \text { (all at COGCEHR) }\end{array}$ & Mukerjee, 1953 \\
\hline 213. & Eria javanica (Sw.) Blume & $\mathrm{E}$ & $\begin{array}{l}\text { Herbarium not available. Live } \\
\text { plants under cultivation. }\end{array}$ & $\begin{array}{l}\text { Shukla, Baishya, } \\
1979\end{array}$ \\
\hline 214. & Eria lasiopetala (Willd.) Ormerod & $\mathrm{E}$ & $\begin{array}{l}\text { Herbarium not available. Live } \\
\text { plants under cultivation. }\end{array}$ & Rao, 2007 \\
\hline 215. & Eria scabrilinguis Lindl. & $\mathrm{E}$ & $\begin{array}{l}\text { Nanda 396, } 833 \text { (all at } \\
\text { COGCEHR) }\end{array}$ & Nanda et al., 2013a \\
\hline 216. & Eria sutepensis Downie & $\mathrm{E}$ & $\begin{array}{l}\text { Nanda 00316; Nanda et al. } 0075 \\
\text { (all at COGCEHR) }\end{array}$ & Phukan, Mao, 2002a \\
\hline 217. & $\begin{array}{l}\text { Eria tomentosa (J. Koenig) Hook. } \\
\text { f. }\end{array}$ & $\mathrm{E}$ & Nanda et al. 00262 (COGCEHR) & $\begin{array}{l}\text { First time report in } \\
\text { present paper }\end{array}$ \\
\hline 218. & Eria vittata Lindl. & $\mathrm{E}$ & $\begin{array}{l}\text { Herbarium not available. Live } \\
\text { plants under cultivation. }\end{array}$ & Hooker, 1890 \\
\hline 219. & Eriodes barbata (Lindl.) Rolfe & $\mathrm{E}$ & $\begin{array}{l}\text { Kishor et al. 00008, Nanda et al. } \\
00088 \text { (all at COGCEHR) }\end{array}$ & Nanda et al., 2013c \\
\hline 220. & Erythrodes blumei (Lindl.) Schltr. & $\mathrm{T}$ & $\begin{array}{l}\text { Inbeng s. n. (acc. no. 590) } \\
\text { (COGCEHR) }\end{array}$ & Nanda et al., 2014c \\
\hline 221. & $\begin{array}{l}\text { Erythrodes hirsuta (Griff.) } \\
\text { Ormerod }\end{array}$ & $\mathrm{T}$ & V. Kumar 1673 (COGCEHR) & Kumar et al., 2016b \\
\hline 222. & $\begin{array}{l}\text { Eulophia bicallosa (D. Don) } \\
\text { Summerh. }\end{array}$ & $\mathrm{T}$ & $\begin{array}{l}\text { G. Watt 6243; S. K. Mukerjee } \\
3023 \text { (all at CAL) }\end{array}$ & Hooker, 1890 \\
\hline 223. & Eulophia dabia (D. Don) Hochr. & $\mathrm{T}$ & S. K. Mukerjee 2717 (CAL) & Mukerjee, 1953 \\
\hline 224. & $\begin{array}{l}\text { Eulophia mannii (Rchb. f.) Hook. } \\
\text { f. }\end{array}$ & $\mathrm{T}$ & Prazer $155(\mathrm{CAL})$ & $\begin{array}{l}\text { A. S. Rao, P. K. } \\
\text { Hajra } 1974\end{array}$ \\
\hline 225 & Eulophia nuda Lindl. & $\mathrm{T}$ & N. L. Bor 17986 (ASSAM) & Chauhan, 2001 \\
\hline 226. & $\begin{array}{l}\text { Eulophia zollingeri (Rchb. f.) J. } \\
\text { J. Sm. }\end{array}$ & $\mathrm{M}$ & $\begin{array}{l}\text { Akimpou 00531; V. Kumar s. n. } \\
\text { (all at COGCEHR); }\end{array}$ & Nanda et al., 2014a \\
\hline 227. & Galeola falconeri Hook. f. & $\mathrm{M}$ & Herbarium not available & Ramakantha, 1995 \\
\hline 228. & $\begin{array}{l}\text { Galeola lindleyana (Hook. f. et } \\
\text { Thomson) Rchb. f. }\end{array}$ & $\mathrm{M}$ & S. K. Mukerjee 2917 (CAL) & Chauhan, 2001 \\
\hline 229. & $\begin{array}{l}\text { Gastrochilus acutifolius (Lindl.) } \\
\text { Kuntze }\end{array}$ & $\mathrm{E}$ & V. Kumar s. n. (COGCEHR) & Ghatak, Devi, 1986 \\
\hline 230. & $\begin{array}{l}\text { Gastrochilus bellinus (Rchb. f.) } \\
\text { Kuntze }\end{array}$ & $\mathrm{E}$ & Herbarium not available & Devi, Ghatak, 1984 \\
\hline 231. & $\begin{array}{l}\text { Gastrochilus calceolaris (Sm.) D. } \\
\text { Don }\end{array}$ & $\mathrm{E}$ & $\begin{array}{l}\text { Nanda et al. s. n. (acc. no. 586) } \\
\text { [COGCEHR] }\end{array}$ & Devi, Ghatak, 1984 \\
\hline 232. & $\begin{array}{l}\text { Gastrochilus distichus (Lindl.) } \\
\text { Kuntze }\end{array}$ & $\mathrm{E}$ & G. Watt 6236,6338 (all at CAL) & Chauhan, 2001 \\
\hline 233. & $\begin{array}{l}\text { Gastrochilus inconspicuous } \\
\text { (Hook. f.) Kuntze }\end{array}$ & $\mathrm{E}$ & C. Sathish Kumar 28740 (TBGT) & Kumar, Kumar, 2005 \\
\hline 234. & $\begin{array}{l}\text { Geodorum densiflorum (Lamk.) } \\
\text { Schltr. }\end{array}$ & $\mathrm{T}$ & S. K. Mukerjee 2949 (CAL) & $\begin{array}{l}\text { Shukla, Baishya, } \\
1979\end{array}$ \\
\hline 235. & $\begin{array}{l}\text { Geodorum recurvum (Roxb.) } \\
\text { Alston }\end{array}$ & $\mathrm{T}$ & Herbarium not available & Mukerjee, 1953 \\
\hline
\end{tabular}


Continuation of table

\begin{tabular}{|c|c|c|c|c|}
\hline $\begin{array}{c}\text { S. } \\
\text { No. }\end{array}$ & Species/variety & Habit & Voucher specimens record & $\begin{array}{l}\text { Reference of first } \\
\text { report for the state }\end{array}$ \\
\hline 236. & Goodyera foliosa (Lindl.) Clarke & $\mathrm{T}$ & G. Watt 0923 (CAL) & Mukerjee, 1953 \\
\hline 237. & $\begin{array}{l}\text { Goodyera procera (Ket-Gawl.) } \\
\text { Hook. }\end{array}$ & $\mathrm{T}$ & Herbarium not available & Clarke, 1889 \\
\hline 238. & $\begin{array}{l}\text { Goodyera schlechtendaliana } \\
\text { Rchb. f. }\end{array}$ & $\mathrm{T}$ & Herbarium not available & Hooker, 1890 \\
\hline 239. & $\begin{array}{l}\text { Goodyera viridiflora (Blume) } \\
\text { Dietrich }\end{array}$ & $\mathrm{T}$ & $\begin{array}{l}\text { V. Kumar 1654, } 1655 \\
\text { (COGCEHR) }\end{array}$ & Kumar, Rao, 2015a \\
\hline 240. & Habenaria acuifera Lindl. & $\mathrm{T}$ & S. K. Mukerjee 3461 (CAL) & Mukerjee, 1953 \\
\hline 241. & Habenaria arietina Hook. f. & $\mathrm{T}$ & S. K. Mukerjee 3268 (CAL) & Mukerjee, 1953 \\
\hline 242. & Habenaria dentata (Sw.) Schltr. & $\mathrm{T}$ & $\begin{array}{l}\text { Akimpou s. n. (acc. no. 408); } \\
\text { K. Chowlu, s. n. (acc. n. 401, } \\
\text { 412) [all at COGCEHR]. S. K. } \\
\text { Mukerjee 3555; A. Meebold } 6404 \\
\text { (all at CAL) }\end{array}$ & Mukerjee, 1953 \\
\hline 243. & $\begin{array}{l}\text { Habenaria malintana (Blanco) } \\
\text { Merl. }\end{array}$ & $\mathrm{T}$ & S. K. Mukerjee 3523(CAL) & Mukerjee, 1953 \\
\hline 244. & $\begin{array}{l}\text { Habenaria mandersii Collet. et } \\
\text { Hemsl. }\end{array}$ & $\mathrm{T}$ & Herbarium not available & Chauhan, 2001 \\
\hline 245. & $\begin{array}{l}\text { Habenaria pelorioides C. S. P. } \\
\text { Parish et Rchb. f. }\end{array}$ & $\mathrm{T}$ & S. K. Mukerjee 3523 (CAL) & Mukerjee, 1953 \\
\hline 246. & $\begin{array}{l}\text { Habenaria reniformis (D. Don) } \\
\text { Hook. f. }\end{array}$ & $\mathrm{T}$ & S. K. Mukerjee 3529 (CAL) & Mukerjee, 1953 \\
\hline 247. & Habenaria trichosantha Lindl. & $\mathrm{T}$ & D. B. Deb 787 (CAL) & Deb, 1961 \\
\hline 248. & Herminium lanceum (Sw.) Vujik & $\mathrm{T}$ & A. A. Mao 106330 (ASSAM). & Mukerjee, 1953 \\
\hline 249. & $\begin{array}{l}\text { Holcoglossum amesianum (Rchb. } \\
\text { f.) Christ. }\end{array}$ & $\mathrm{E}$ & $\begin{array}{l}\text { Herbarium not available. Live } \\
\text { plants under cultivation. }\end{array}$ & Devi, Ghatak, 1984 \\
\hline 250. & $\begin{array}{l}\text { Holcoglossum himalaicum (Deb } \\
\text { et al.) Aver. }\end{array}$ & $\mathrm{E}$ & $\begin{array}{l}\text { Kishor et al. 00458, } 00256 \text { (all at } \\
\text { COGCEHR) }\end{array}$ & Chauhan, 2001 \\
\hline 251. & $\begin{array}{l}\text { Liparis bistriata C. S. P. Parish et } \\
\text { Rchb. f. }\end{array}$ & $\mathrm{E}$ & S. K. Mukerjee 2881(CAL) & Mukerjee, 1953 \\
\hline 252. & Liparis bootanensis Griff. & $\mathrm{E}$ & $\begin{array}{l}\text { Herbarium not available. Live } \\
\text { plants under cultivation. }\end{array}$ & Chauhan, 2001 \\
\hline 253. & Liparis caespitosa (Lam.) Lindl. & E & $\begin{array}{l}\text { Chowlu 528; Kishor et al. s. n. } \\
\text { (acc.no. 497) [all at COGCEHR] }\end{array}$ & Kumar, Kumar, 2005 \\
\hline 254. & Liparis distans Clarke & $\mathrm{E}$ & S. K. Mukerjee 3540 (CAL). & Mukerjee, 1953 \\
\hline 255. & Liparis duthiei Hook. f. & $\mathrm{E}$ & $\begin{array}{l}\text { Chowlu s. n. (accc.no. 236) } \\
\text { [COGCEHR] }\end{array}$ & $\begin{array}{l}\text { First time report in } \\
\text { present paper }\end{array}$ \\
\hline 256. & Liparis elliptica Wight & $\mathrm{E}$ & Chowlu 00428 (COGEHR). & $\begin{array}{l}\text { Chowlu et al., 2014; } \\
\text { Rao, } 2015\end{array}$ \\
\hline 257. & Liparis odorata (Willd.) Lindl. & $\mathrm{T}$ & $\begin{array}{l}\text { V. Kumar } 1664 \text { (COGCEHR). S. } \\
\text { K. Mukerjee 2286, } 3258 \text { (CAL) }\end{array}$ & Mukerjee, 1953 \\
\hline 258. & Liparis plantaginea Lindl. & $\mathrm{E}$ & Chowlu 722 (COGCEHR) & Chauhan, 2001 \\
\hline 259. & Liparis resupinata Ridl. & $\mathrm{E}$ & Herbarium not available & Chauhan, 2001 \\
\hline 260. & Liparis spathulata Lindl. & $\mathrm{E}$ & $\begin{array}{l}\text { Herbarium not available. Live } \\
\text { plants under cultivation. }\end{array}$ & Clarke, 1889 \\
\hline 261. & Liparis viridiflora (Blume) Lindl. & $\mathrm{E}$ & $\begin{array}{l}\text { Imbeng s. n. (acc. no. 421); } \\
\text { Nanda s. n. (acc. no. 98) [all at } \\
\text { COGCEHR)]. A. Meebold } 6482 \\
\text { (CAL) }\end{array}$ & Clarke, 1889 \\
\hline
\end{tabular}




\section{Continuation of table}

\begin{tabular}{|c|c|c|c|c|}
\hline $\begin{array}{c}\text { S. } \\
\text { No. }\end{array}$ & Species/variety & Habit & Voucher specimens record & $\begin{array}{l}\text { Reference of first } \\
\text { report for the state }\end{array}$ \\
\hline 262. & Luisia antennifera Blume & $\mathrm{E}$ & Herbarium not available & Ghatak, Devi, 1986 \\
\hline 263. & Luisia filiformis Hook. $\mathrm{f}$. & $\mathrm{E}$ & $\begin{array}{l}\text { Nanda et al. 00010, } 00087 \text { (all at } \\
\text { COGCEHR) }\end{array}$ & Nanda et al., 2013c \\
\hline 264. & Luisia jonesii J. J. Sm. & $\mathrm{E}$ & Herbarium not available & Ghatak, Devi, 1986 \\
\hline 265. & Luisia trichorhiza (Hook.) Blume & $\mathrm{E}$ & $\begin{array}{l}\text { Nanda (acc. No. 988), } \\
\text { [COGCEHR] }\end{array}$ & $\begin{array}{l}\text { First time report in } \\
\text { present paper }\end{array}$ \\
\hline 266. & $\begin{array}{l}\text { Mycaranthes floribunda (D. Don) } \\
\text { Chen et Wood }\end{array}$ & $\mathrm{E}$ & $\begin{array}{l}\text { Nanda et al. s. n. (acc. no. 593) } \\
\text { [COGCEHR] }\end{array}$ & Mao, 1999 \\
\hline 267. & $\begin{array}{l}\text { Mycaranthes pannea (Lindl.) } \\
\text { Chen et Wood }\end{array}$ & $\mathrm{E}$ & $\begin{array}{l}\text { Kishor et al. s. n. (acc. no. 322) } \\
\text { [COGCEHR] }\end{array}$ & Ghatak, Devi, 1986 \\
\hline 268. & $\begin{array}{l}\text { Myrmechis pumila (Hook. f.) } \\
\text { Tang et Wang }\end{array}$ & $\mathrm{T}$ & Capt. K. Ward 3267 (CAL) & Mukerjee, 1953 \\
\hline 269. & $\begin{array}{l}\text { Neogyna gardneriana (Lindl.) } \\
\text { Rchb. f. }\end{array}$ & $\mathrm{T}$ & G. Watt 5871 (CAL) & Chauhan, 2001 \\
\hline 270. & $\begin{array}{l}\text { Nephelaphyllum pulchrum } \\
\text { Blume }\end{array}$ & $\mathrm{T}$ & Herbarium not available & Kumar, Kumar, 2005 \\
\hline 271. & Nervilia concolor (Blume) Schltr. & $\mathrm{T}$ & Herbarium not available & Chauhan, 2001 \\
\hline 272. & $\begin{array}{l}\text { Nervilia crociformis (Zoll. et } \\
\text { Mor.) Seidenf. }\end{array}$ & $\mathrm{T}$ & D. B. Deb 2497, 2527 (CAL) & Mukerjee, 1953 \\
\hline 273. & $\begin{array}{l}\text { Nervilia falcata (King et Pantl.) } \\
\text { Schltr. }\end{array}$ & $\mathrm{T}$ & Herbarium not available & Chauhan, 2001 \\
\hline 274. & Nervilia plicata (Andr.) Schltr. & $\mathrm{T}$ & $\begin{array}{l}\text { Herbarium not available. Live } \\
\text { plants under cultivation. }\end{array}$ & Chauhan, 2001 \\
\hline 275. & Oberonia acaulis Griff. & $\mathrm{E}$ & $\begin{array}{l}\text { Meehold } 6642 \text { (MH), Mao } 106405 \\
\text { (ASSAM); V. Kumar } 1684 \\
\text { (COGCEHR) }\end{array}$ & Mao, 1999 \\
\hline 276. & $\begin{array}{l}\text { Oberonia acaulis Griff. var. } \\
\text { latipetala Chowlu et al. }\end{array}$ & $\mathrm{E}$ & Chowlu 00368 (COGCEHR) & Chowlu et al., 2014b \\
\hline 277. & Oberonia bicornis Lindl. & $\mathrm{E}$ & A. Mao 101966 (ASSAM) & Mao, 1999 \\
\hline 278. & Oberonia clarkeii Hook. f. & $\mathrm{E}$ & Herbarium not available & Chauhan, 2001 \\
\hline 279. & Oberonia ensiformis (Sm.) Lindl. & $\mathrm{E}$ & $\begin{array}{l}\text { Chowlu s. n. (acc. n. 423) } \\
\text { [COGCEHR] }\end{array}$ & Chauhan, 2001 \\
\hline 280. & Oberonia integerrima Guill. & $\mathrm{E}$ & Kipgen 00334 (COGCEHR) & Chowlu et al., 2013b \\
\hline 281. & Oberonia jenkinsiana Lindl. & $\mathrm{E}$ & $\begin{array}{l}\text { V. Kumar 1659; Inbeng and Atan } \\
\text { 00012; Chowlu } 00486 \text { (all at } \\
\text { COGCEHR) }\end{array}$ & Chowlu et al., 2012b \\
\hline 282. & Oberonia longibracteata Lindl. & $\mathrm{E}$ & A. Mao 101940 (ASSAM) & Mao, 1999 \\
\hline 283. & $\begin{array}{l}\text { Oberonia manipurensis Chowlu } \\
\text { et al. }\end{array}$ & $\mathrm{E}$ & $\begin{array}{l}\text { K. Chowlu } 00362 \text { (CAL). K. } \\
\text { Chowlu } 00441 \text { (COGCEHR) }\end{array}$ & Chowlu et al., $2015 \mathrm{a}$ \\
\hline 284. & Oberonia maxima Hook. f. & $\mathrm{E}$ & $\begin{array}{l}\text { Chowlu 00123, } 00388 \text { (all at } \\
\text { COGCEHR) }\end{array}$ & Deb, 1961 \\
\hline 285. & $\begin{array}{l}\text { Oberonia mucronata (D. Don) } \\
\text { Ormerod et Seidenf. }\end{array}$ & $\mathrm{E}$ & Chowlu 00425 (COGCEHR) & Chauhan, 2001 \\
\hline 286. & Oberonia myosurus Lindl. & $\mathrm{E}$ & A. Mao 101971 (ASSAM) & Mao, 1999 \\
\hline 287. & Oberonia pachyrachis Rchb. f. & $\mathrm{E}$ & $\begin{array}{l}\text { Chowlu s. n. (acc. no. 91) } \\
\text { [COGCEHR] }\end{array}$ & Devi et al., 2018 \\
\hline 288. & $\begin{array}{l}\text { Oberonia pyrulifera King et } \\
\text { Pantl. }\end{array}$ & $\mathrm{E}$ & $\begin{array}{l}\text { G. Watt } 6438 ; \text { S. K. Mukerjee } \\
2880 \text { (all at CAL) }\end{array}$ & Hooker, 1890 \\
\hline
\end{tabular}


Continuation of table

\begin{tabular}{|c|c|c|c|c|}
\hline $\begin{array}{l}\text { S. } \\
\text { No. }\end{array}$ & Species/variety & Habit & Voucher specimens record & $\begin{array}{l}\text { Reference of first } \\
\text { report for the state }\end{array}$ \\
\hline 289. & Oberonia teres Kerr & $\mathrm{E}$ & Chowlu 00358 (COGCEHR). & Phukan, Mao, 2002a \\
\hline 290. & $\begin{array}{l}\text { Odontochilus grandiflorus } \\
\text { (Lindl.) Hook. f. }\end{array}$ & $\mathrm{T}$ & Herbarium not available & Chauhan, 2001 \\
\hline 291. & $\begin{array}{l}\text { Odontochilus tetrapterus (Hook. } \\
\text { f.) Bhatta. et Chowdh. }\end{array}$ & $\mathrm{T}$ & C. B. Clarke 42191 (K) & Hooker, 1890 \\
\hline 292. & Otochilus albus Lindl. & $\mathrm{E}$ & $\begin{array}{l}\text { D. B. Deb } 1435,1892 ; \text { S. K. } \\
\text { Mukerjee } 3039 \text { (all at CAL) }\end{array}$ & Mukerjee, 1953 \\
\hline 293. & Otochilus fuscus Lindl. & $\mathrm{E}$ & $\begin{array}{l}\text { K. Chowlu s. n. (acc. no. 344, } \\
\text { 345); Kishor 11190, 00194, 00197 } \\
\text { (all at COGCEHR); G. Watt 6482; } \\
\text { A. Meebold } 7105 \text { (CAL) }\end{array}$ & Hooker, 1890 \\
\hline 294. & Otochilus porrectus Lindl. & $\mathrm{E}$ & Herbarium not available & Hooker, 1890 \\
\hline 295. & Pachystoma pubescens Blume & $\mathrm{T}$ & $\begin{array}{l}\text { Biswajit et al. s. n. (acc. no. 343) } \\
\text { [COGCEHR] }\end{array}$ & Hooker, 1890 \\
\hline 296. & Panisea apiculata Lindl. & $\mathrm{E}$ & Biswajeet WL 194 (COGCEHR) & $\begin{array}{l}\text { First time report in } \\
\text { present paper }\end{array}$ \\
\hline 297. & Panisea demissa (D. Don) Pfitz. & $\mathrm{E}$ & Biswajit s. n. 235 (COGCEHR) & Kumar, Kumar, 2005 \\
\hline 298. & Panisea tricallosa Rolfe & $\mathrm{E}$ & $\begin{array}{l}\text { J. Devi } 2351 \text { (Manipur Univ. } \\
\text { Herbarium) }\end{array}$ & Chauhan, 2001 \\
\hline 299. & Panisea uniflora (Lindl.) Lindl. & $\mathrm{E}$ & $\begin{array}{l}\text { COGCEHR 00105, Chowlu } 00172 \\
\text { (all at COGCEHR) }\end{array}$ & Chowlu et al., 2012a \\
\hline 300. & $\begin{array}{l}\text { Paphiopedilum hirsutissimum } \\
\text { (Hook.) Stein }\end{array}$ & $\mathrm{T}$ & $\begin{array}{l}\text { S. coll. } 340 \text { (COGCEHR); J. } \\
\text { Devi } 2322 \text { (Manipur University } \\
\text { Herbarium) }\end{array}$ & Devi, Ghatak, 1984 \\
\hline 301. & $\begin{array}{l}\text { Paphiopedilum insigne (Lindl.) } \\
\text { Pfitz. }\end{array}$ & $\mathrm{T}$ & $\begin{array}{l}\text { J. Devi } 2356 \text { (Manipur Univ. } \\
\text { Herbarium) }\end{array}$ & Devi, Ghatak, 1984 \\
\hline 302. & $\begin{array}{l}\text { Paphiopedilum spicerianum } \\
\text { (Rchb. f.) Pfitz. }\end{array}$ & $\mathrm{T}$ & $\begin{array}{l}\text { J. Devi } 2457 \text { (Manipur Univ. } \\
\text { Herbarium) }\end{array}$ & Devi, Ghatak, 1984 \\
\hline 303. & $\begin{array}{l}\text { Paphiopedilum venustum (Wall.) } \\
\text { Stein }\end{array}$ & $\mathrm{T}$ & $\begin{array}{l}\text { J. Devi } 2339 \text { (Manipur Univ. } \\
\text { Herbarium) }\end{array}$ & Devi, Ghatak, 1984 \\
\hline 304. & $\begin{array}{l}\text { Paphiopedilum villosum (Lindl.) } \\
\text { Stein }\end{array}$ & $\mathrm{T}$ & $\begin{array}{l}\text { J. Devi } 2358 \text { (Manipur Univ. } \\
\text { Herbarium) }\end{array}$ & Devi, Ghatak, 1984 \\
\hline 305. & $\begin{array}{l}\text { Papilionanthe teres (Roxb.) } \\
\text { Schltr. }\end{array}$ & $\mathrm{E}$ & C. Sathish Kumar s. n. (TBGT) & Chauhan, 2001 \\
\hline 306. & $\begin{array}{l}\text { Papilionanthe uniflora (Lindl.) } \\
\text { Garay }\end{array}$ & $\mathrm{E}$ & Nanda et al. 00380 (COGCEHR) & Nanda et al., 2014c \\
\hline 307. & $\begin{array}{l}\text { Papilionanthe vandarum (Rchb. } \\
\text { f.) Garay }\end{array}$ & $\mathrm{E}$ & S. K. Mukerjee 6290 (CAL). & Clarke, 1889 \\
\hline 308. & Pecteilis henryii Schltr. & $\mathrm{T}$ & N. L. Bor 18495 (DD). & Mukerjee, 1953 \\
\hline 309. & Pecteilis susannae (L.) Rafin. & $\mathrm{T}$ & S. K. Mukerjee 3463 (CAL) & Chauhan, 2001 \\
\hline 310. & $\begin{array}{l}\text { Pelatantheria insectifera (Rchb. } \\
\text { f.) Ridl. }\end{array}$ & $\mathrm{T}$ & Akimpou 00529 (COGCEHR) & Nanda et al., 2014d \\
\hline 311. & $\begin{array}{l}\text { Peristylus affinis (D. Don) } \\
\text { Seidenf. }\end{array}$ & $\mathrm{T}$ & Herbarium not available & Chauhan, 2001 \\
\hline 312. & $\begin{array}{l}\text { Peristylus densus(Lindl.) Sant. et } \\
\text { Kapad. }\end{array}$ & $\mathrm{T}$ & S. K. Mukerjee 3202 (CAL) & Mukerjee, 1953 \\
\hline 313. & $\begin{array}{l}\text { Peristylus goodyeroides (D. Don) } \\
\text { Lindl. }\end{array}$ & $\mathrm{T}$ & Deb. 1005 (CAL) & Deb, 1961 \\
\hline 314. & $\begin{array}{l}\text { Peristylus lacertiferus (Lindl.) J. } \\
\text { J. Sm. }\end{array}$ & $\mathrm{T}$ & V. Kumar 1663 (COGCEHR) & Deb, 1961 \\
\hline
\end{tabular}




\section{Continuation of table}

\begin{tabular}{|c|c|c|c|c|}
\hline $\begin{array}{c}\text { S. } \\
\text { No. }\end{array}$ & Species/variety & Habit & Voucher specimens record & $\begin{array}{l}\text { Reference of first } \\
\text { report for the state }\end{array}$ \\
\hline 315. & $\begin{array}{l}\text { Peristylus mannii (Rchb. f.) } \\
\text { Mukerj. }\end{array}$ & $\mathrm{T}$ & S. K. Mukerjee 3731 (CAL) & Mukerjee, 1953 \\
\hline 316. & Peristylus parishii Rchb. f. & $\mathrm{T}$ & $\begin{array}{l}\text { V. Kumar 1667; Chowlu } 00957 \\
\text { (all at COGCEHR) }\end{array}$ & Chowlu et al., 2014c \\
\hline 317. & Peristylus richardianus Wight & $\mathrm{T}$ & Chowlu 00954 (COGCEHR) & Chowlu et al., 2014c \\
\hline 318. & $\begin{array}{l}\text { Peristylus tipuliferus (C. S. P. } \\
\text { Parish et Rchb. f.) Mukerj. }\end{array}$ & $\mathrm{T}$ & S. K. Mukerjee 3364, 3417 (CAL) & Mukerjee, 1953 \\
\hline 319. & Phaius flavus (Blume) Lindl. & $\mathrm{T}$ & S. K. Mukerjee 2937 (CAL) & Mukerjee, 1953 \\
\hline 320. & $\begin{array}{l}\text { Phaius mishmensis (Lindl. et } \\
\text { Paxt.) Rchb. f. }\end{array}$ & $\mathrm{T}$ & Herbarium not available & Devi, Ghatak, 1984 \\
\hline 321. & $\begin{array}{l}\text { Phaius tankervilliae (Banks) } \\
\text { Blume }\end{array}$ & $\mathrm{T}$ & Nanda et al. 274 (COGCEHR) & Mukerjee, 1953 \\
\hline 322. & Phaius wallichii Lindl. & $\mathrm{T}$ & $\begin{array}{l}\text { S. K. Mukerjee } 2457 \text { (CAL); } \\
\text { Herbarium not available. Live } \\
\text { plants under cultivation. }\end{array}$ & $\begin{array}{l}\text { Laiphrakpam et al., } \\
2015\end{array}$ \\
\hline 323. & $\begin{array}{l}\text { Phalaenopsis cornu-cervi (Breda) } \\
\text { Blume et Rchb. f. }\end{array}$ & $\mathrm{E}$ & $\begin{array}{l}\text { J. Devi } 2378 \text { (Manipur Univ. } \\
\text { Herbarium) }\end{array}$ & Devi, Ghatak, 1984 \\
\hline 324. & $\begin{array}{l}\text { Phalaenopsis difformis (Lindl.) } \\
\text { Kocyan et Schuit. }\end{array}$ & $\mathrm{E}$ & $\begin{array}{l}\text { Herbarium not available. Live } \\
\text { plants under cultivation. }\end{array}$ & Chauhan, 2001 \\
\hline 325. & Phalaenopsis fasciata Rchb. f. & $\mathrm{E}$ & $\begin{array}{l}\text { C. Sathish Kumar 28259, } 28764 \\
\text { (all at TBGT). }\end{array}$ & Kumar, Kumar, 2005 \\
\hline 326. & $\begin{array}{l}\text { Phalaenopsis hygrochila J. M. H. } \\
\text { Shaw }\end{array}$ & $\mathrm{E}$ & $\begin{array}{l}\text { Nanda et al. } 540 \text { (COGCEHR). S. } \\
\text { K. Mukerjee } 2946 \text { (CAL) }\end{array}$ & Mukerjee, 1953 \\
\hline 327. & $\begin{array}{l}\text { Phalaenopsis pulcherrima } \\
\text { (Lindl.) J. J. Sm. }\end{array}$ & $\mathrm{E}$ & Mao 101970 (ASSAM) & Chauhan et al., 2001 \\
\hline 328. & $\begin{array}{l}\text { Phalaenopsis taenialis (Lindl.) } \\
\text { Christen. }\end{array}$ & E & Kishor et al. 00009 (COGCEHR) & Kumar, Kumar, 2005 \\
\hline 329. & $\begin{array}{l}\text { Phalaenopsis yingjiangensis (Z. } \\
\text { H. Tsi) Kocyan et Schuit. }\end{array}$ & $\mathrm{E}$ & V. Kumar 1677 (COGCEHR) & Kumar et al., 2016b \\
\hline 330. & Pholidota articulata Lindl. & $\mathrm{E}$ & $\begin{array}{l}\text { Kishor 00070B; Chowlu 00029, } \\
00080 \text { (all at COGCEHR); S. K. } \\
\text { Mukerjee 3212; A. Meebold 5525, } \\
7080 \text { (all at CAL) }\end{array}$ & Mukerjee, 1953 \\
\hline 331. & $\begin{array}{l}\text { Pholidota convallariae (C. S. P. } \\
\text { Parish et Rchb. f.) Hook. f. }\end{array}$ & $\mathrm{E}$ & Chowlu 502, 503 (COGCEHR) & Chowlu, Rao, 2015b \\
\hline 332. & Pholidota imbricataHook. & $\mathrm{E} / \mathrm{L}$ & $\begin{array}{l}\text { Chowlu 0028, 0074, } 0079 \text { (all at } \\
\text { COGCEHR); S. C. Sinha 1890; } \\
\text { S. K. Mukerjee 2942; A. Meebold } \\
6856 \text { (CAL) }\end{array}$ & Clarke, 1889 \\
\hline 333. & Pholidota pallida Lindl. & $\mathrm{E}$ & $\begin{array}{l}\text { Chowlu s. n. (acc. no. 404); } \\
\text { Chowlu } 00085 \text { [all at COGCEHR] }\end{array}$ & Chowlu, Rao, 2015b \\
\hline 334. & Pholidota protracta Hook. f. & $\mathrm{E} / \mathrm{L}$ & S. K. Mukerjee 3722 (CAL) & Mukerjee, 1953 \\
\hline 335. & $\begin{array}{l}\text { Pholidota pygmaea H. J. Chowd- } \\
\text { hery et G. D. Pal }\end{array}$ & $\mathrm{E}$ & Chowlu 00392 (COGCEHR) & Chowlu et al., 2013d \\
\hline 336. & Pholidota rubraLindl. & $\mathrm{E}$ & $\begin{array}{l}\text { Chowlu 00179, } 00178 \\
\text { (COGCEHR). A. Meebold } 7073 \\
\text { (CAL) }\end{array}$ & $\begin{array}{l}\text { First time report in } \\
\text { present paper }\end{array}$ \\
\hline 337. & Phreatia elegans Lindl. & $\mathrm{E}$ & Nanda et al. 00055 (COGCEHR) & Mao, 1999 \\
\hline 338. & Pinalia acervata (Lindl.) Kuntze & $\mathrm{E}$ & $\begin{array}{l}\text { Kishor et al. 00279; Nanda et al. } \\
303 \text { (all at COGCEHR) }\end{array}$ & Mao, 1999 \\
\hline
\end{tabular}


Continuation of table

\begin{tabular}{|c|c|c|c|c|}
\hline $\begin{array}{l}\text { S. } \\
\text { No. }\end{array}$ & Species/variety & Habit & Voucher specimens record & $\begin{array}{l}\text { Reference of first } \\
\text { report for the state }\end{array}$ \\
\hline 339. & Pinalia amica (Rchb. f.) Kuntze & $\mathrm{E}$ & Nanda et al. 00455 (COGCEHR) & Mao, 1999 \\
\hline 340. & Pinalia excavata (Lindl.) Kuntze & $\mathrm{E}$ & S. K. Mukerjee 3237(CAL) & Mukerjee, 1953 \\
\hline 341. & $\begin{array}{l}\text { Pinaliaglobulifera (Seidenf.) A. } \\
\text { N. Rao }\end{array}$ & $\mathrm{E}$ & $\begin{array}{l}\text { C. Sathish Kumar 28748, } 27362 \\
\text { (all at TBGT) }\end{array}$ & Kumar, Kumar, 2005 \\
\hline 342. & $\begin{array}{l}\text { Pinalia spicata (D. Don) Chen et } \\
\text { Wood }\end{array}$ & $\mathrm{E}$ & $\begin{array}{l}\text { V. Kumar 1685; Nanda et al. } \\
00461,00462 \text { (all at COGCEHR) }\end{array}$ & Chauhan, 2001 \\
\hline 343. & Pinalia stricta (Lindl.) Kuntze & $\mathrm{E}$ & Nanda et al. 00053 (COGCEHR) & Nanda et al., 2013c \\
\hline 344. & $\begin{array}{l}\text { Pleione hookeriana (Lindl.) } \\
\text { Rollisson }\end{array}$ & $\mathrm{E}$ & S. K. Mukerjee 3238 (CAL) & Mukerjee, 1953 \\
\hline 345. & Pleione humilis (Sm.) D. Don & E & $\begin{array}{l}\text { Chowlu 1569, } 1570 \text { (all at } \\
\text { COGCEHR) }\end{array}$ & Chauhan, 2001 \\
\hline 346. & $\begin{array}{l}\text { Pleione maculata (Lindl.) Lindl. } \\
\text { et Paxton }\end{array}$ & $\mathrm{E}$ & $\begin{array}{l}\text { Chowlu } 534 \text { (COGCEHR); A. } \\
\text { Meebold } 6562 \text { (CAL) }\end{array}$ & Chauhan, 2001 \\
\hline 347. & Pleione praecox (Sm.) D. Don & $\mathrm{E}$ & $\begin{array}{l}\text { Chowlu 00535, } 00543 \text { (all at } \\
\text { COGCEHR); S. K. Mukerjee } 3655 \\
\text { (CAL) }\end{array}$ & Muk \\
\hline 348. & $\begin{array}{l}\text { Polystachya concreta (Jacq.) } \\
\text { Garay et H. R. Sweet }\end{array}$ & $\mathrm{E}$ & $\begin{array}{l}\text { Kishor s. n. (acc. no. 377), } \\
\text { Chowlu s. n. (acc. no. 385) [all at } \\
\text { COGCEHR] }\end{array}$ & Kumar, Kumar, 2005 \\
\hline 349. & $\begin{array}{l}\text { Ponerorchis secundiflora } \\
\text { (Kraenzl.) X. H. Jin et al. }\end{array}$ & $\mathrm{T}$ & Herbarium not available & Chauhan, 2001 \\
\hline 350. & Porpax gigantea Deori & $\mathrm{E}$ & Herbarium not available & Deori, 1978 \\
\hline 351. & Pteroceras teres (Blume) Holtt. & $\mathrm{E}$ & D. B. Deb 1896 (CAL) & $\begin{array}{l}\text { First time report in } \\
\text { present paper }\end{array}$ \\
\hline 352. & Renanthera imschootiana Rolfe & $\mathrm{E}$ & $\begin{array}{l}\text { Nanda et al. 00385, } 00281 \text { (all at } \\
\text { COGCEHR) }\end{array}$ & $\begin{array}{l}\text { Shukla, Baishya, } \\
1979\end{array}$ \\
\hline 353. & $\begin{array}{l}\text { Rhynchostylis gigantea (Lindl.) } \\
\text { Ridl. }\end{array}$ & $\mathrm{E}$ & $\begin{array}{l}\text { J. Devi } 2383 \text { (Manipur Univ. } \\
\text { Herbarium) }\end{array}$ & Devi, Ghatak, 1984 \\
\hline 354. & Rhynchostylis retusa (L.) Blume & $\mathrm{E}$ & C. Sathish Kumar 28737 (TBGT) & Devi, Ghatak, 1984 \\
\hline 355. & $\begin{array}{l}\text { Sarcoglyphis manipurensis Rao } \\
\text { et al. }\end{array}$ & $\mathrm{E}$ & $\begin{array}{l}\text { H.B. Sharma 596; Nanda and } \\
\text { Akimpou } 530 \text { (all at COGCEHR). } \\
\text { H.B. Sharma } 596 \text { (CAL) }\end{array}$ & Kumar et al., 2016a \\
\hline 356. & Satyrium nepalense D. Don & $\mathrm{T}$ & S. K. Mukerjee 3392 (CAL) & Mukerjee, 1953 \\
\hline 357. & $\begin{array}{l}\text { Schoenorchis fragrans (C. S. P. } \\
\text { Parish et Rchb. f.) Seidenf. }\end{array}$ & $\mathrm{E}$ & $\begin{array}{l}\text { Chowlu s. n. (acc. no. 367) } \\
\text { (COGCEHR) }\end{array}$ & Devi, Ghatak, 1984 \\
\hline 358. & $\begin{array}{l}\text { Schoenorchis gemmata (Lindl.) } \\
\text { J. J. Sm. }\end{array}$ & E & Nanda et al. 00118 (COGCEHR) & Chauhan, 2001 \\
\hline 359. & $\begin{array}{l}\text { Smitinandia micrantha (Lindl.) } \\
\text { Holtt. }\end{array}$ & $\mathrm{E}$ & Nanda et al. 642 (COGCEHR) & Nanda et al., 2014c \\
\hline 360. & $\begin{array}{l}\text { Spathoglottis pubescens Lindl. } \\
\text { var. parvifolia Hook. f. }\end{array}$ & $\mathrm{T}$ & $\begin{array}{l}\text { V. Kumar 1657; Nanda et al. s. n. } \\
\text { (acc. no. 575) [all at COGCEHR] }\end{array}$ & Mukerjee, 1953 \\
\hline 361. & $\begin{array}{l}\text { Spiranthes himalayensis Survesw. } \\
\text { et al. }\end{array}$ & $\mathrm{T}$ & $\begin{array}{l}\text { S. Surveswaran } 1 \text { (HJCB 1001) } \\
\text { (JCB) }\end{array}$ & $\begin{array}{l}\text { Surveswaran et al., } \\
2017\end{array}$ \\
\hline 362. & Spiranthes sinensis (Pers.) Ames & $\mathrm{T}$ & $\begin{array}{l}\text { S. K. Mukerjee 3732, } 2742 \text { (all at } \\
\text { CAL) }\end{array}$ & Mukerjee, 1953 \\
\hline 363. & $\begin{array}{l}\text { Taeniophyllum glandulosum } \\
\text { Blume }\end{array}$ & E & Kishor 00002 (COGCEHR) & Nanda et al., 2012a \\
\hline 364. & Tainia latifolia (Lindl.) Rchb. f. & $\mathrm{T}$ & D. B. Deb 2583(CAL). & Kumar, Kumar, 2005 \\
\hline
\end{tabular}


End of table

\begin{tabular}{|c|c|c|c|c|}
\hline $\begin{array}{c}\text { S. } \\
\text { No. }\end{array}$ & Species/variety & Habit & Voucher specimens record & $\begin{array}{l}\text { Reference of first } \\
\text { report for the state }\end{array}$ \\
\hline 365. & Thelasis longifolia Hook. f. & $\mathrm{E}$ & $\begin{array}{l}\text { Kipgen } 00540 \text {, Chowlu et al. } 1108 \\
\text { (all at COGCEHR) }\end{array}$ & $\begin{array}{l}\text { Chowlu et al., 2014; } \\
\text { Rao, } 2015\end{array}$ \\
\hline 366. & Thelasis pygmea Lindl. & $\mathrm{E}$ & $\begin{array}{l}\text { Chowlu et al. s. n. (acc. no. 534) } \\
\text { [COGCEHR] }\end{array}$ & Mao, 1999 \\
\hline 367. & Thrixspermum centipeda Lour. & $\mathrm{E}$ & Herbarium not available & Chauhan, 2001 \\
\hline 368. & $\begin{array}{l}\text { Thrixspermum indicum Vik. } \\
\text { Kumar et al. }\end{array}$ & $\mathrm{E}$ & Nanda et al. 00015 (COGCEHR) & Kumar et al., 2017 \\
\hline 369. & Thunia alba (Lindl.) Rchb. f. & $\mathrm{E}$ & $\begin{array}{l}\text { Chowlu 00359, 1201; Kishor } \\
00177 \text { (all at COGCEHR) }\end{array}$ & Mukerjee, 1953 \\
\hline 370. & Tropidia curculigoides Lindl. & $\mathrm{T}$ & S. K. Mukerjee 3702 (CAL) & Mukerjee, 1953 \\
\hline 371. & Tropidia namasiae Liao et al. & $\mathrm{T}$ & V. Kumar 1653 (COGCEHR) & Kumar et al., 2015 \\
\hline 372. & Uncifera acuminata Lindl. & $\mathrm{E}$ & Herbarium not available & Chauhan, 2001 \\
\hline 373. & Uncifera obtusifolia Lindl. & $\mathrm{E}$ & Nanda et al. 318 (COGCEHR) & Nanda et al., 2013c \\
\hline 374. & Vanda alpina Lindl. & $\mathrm{E}$ & A. Mao 101953 (ASSAM) & Mao, 1999 \\
\hline 375. & $\begin{array}{l}\text { Vanda ampullacea (Roxb.) L. M. } \\
\text { Gardiner }\end{array}$ & $\mathrm{E}$ & C. Sathish Kumar 28732 (TBGT) & Clarke, 1889 \\
\hline 376. & Vanda bicolor Griff. & $\mathrm{E}$ & $\begin{array}{l}\text { Nanda 242, } 272 \text { (all at } \\
\text { COGCEHR) }\end{array}$ & Nanda et al., 2014c \\
\hline 377. & Vanda coerulea Lindl. & $\mathrm{E}$ & $\begin{array}{l}\text { Nanda 00070, 00071 (all at } \\
\text { COGCEHR). S. K. Mukerjee } 3480 \\
(\mathrm{CAL})\end{array}$ & Mukerjee, 1953 \\
\hline 378. & Vanda coerulescens Griff. & $\mathrm{E}$ & $\begin{array}{l}\text { N. L. Bor } 18456 \text { (K). C. Sathish } \\
\text { Kumar } 28706 \text { (TBGT). }\end{array}$ & Devi, Ghatak, 1984 \\
\hline 379. & Vanda cristata Lindl. & $\mathrm{E}$ & V. Kumar 1678 (COGCEHR) & Chauhan, 2001 \\
\hline 380. & $\begin{array}{l}\text { Vanda miniata (Lindl.) L. M. } \\
\text { Gardiner }\end{array}$ & $\mathrm{E}$ & A. Mao 101990 (ASSAM) & Phukan, Mao, 2002a \\
\hline 381. & $\begin{array}{l}\text { Vanda motesiana Choltco (sensu } \\
\text { V. stangeana) }\end{array}$ & $\mathrm{E}$ & $\begin{array}{l}\text { Nanda et al. } 00067 \text { (COGCEHR); } \\
\text { Limasenla } 17006 \text { (NEHU) }\end{array}$ & $\begin{array}{l}\text { Choltco, 2009; } \\
\text { Phukan, } 1996\end{array}$ \\
\hline 382. & Vanda pumila Hook. f. & $\mathrm{E}$ & Herbarium not available & Kumar, Kumar, 2005 \\
\hline 383. & Vanda testacea (Lindl.) Rchb. f. & $\mathrm{E}$ & Kishor et al. 240 (COGCEHR) & Devi, Ghatak, 1984 \\
\hline 384. & $\begin{array}{l}\text { Vandopsis undulata (Lindl.) J. J. } \\
\text { Sm. }\end{array}$ & $\mathrm{E}$ & S. K. Mukerjee 2726 (CAL) & Mukerjee, 1953 \\
\hline 385. & Vanilla aphylla Blume & $\mathrm{E}$ & C. Sathish Kumar 28270 (TBGT) & $\begin{array}{l}\text { Chauhan, 2001, } \\
\text { Kumar, Kumar, } 2005\end{array}$ \\
\hline 386. & Yoania japonica Maxim. & $\mathrm{M}$ & Herbarium not available & Hooker, 1890 \\
\hline 387. & Zeuxine affinis (Lindl.) Hook. f. & $\mathrm{T}$ & $\begin{array}{l}\text { V. Kumar 1169, 1170; A. N. } \\
\text { Rao 316; Y. Nanda } 430 \text { (all at } \\
\text { COGCEHR) }\end{array}$ & Nanda et al., 2014c \\
\hline 388. & Zeuxine nervosa (Lindl.) Trimen & $\mathrm{T}$ & Herbarium not available & Clarke, 1889 \\
\hline 389. & Zeuxine strateumatica (L.) Schltr. & $\mathrm{T}$ & Herbarium not available & Rao, 2007 \\
\hline
\end{tabular}




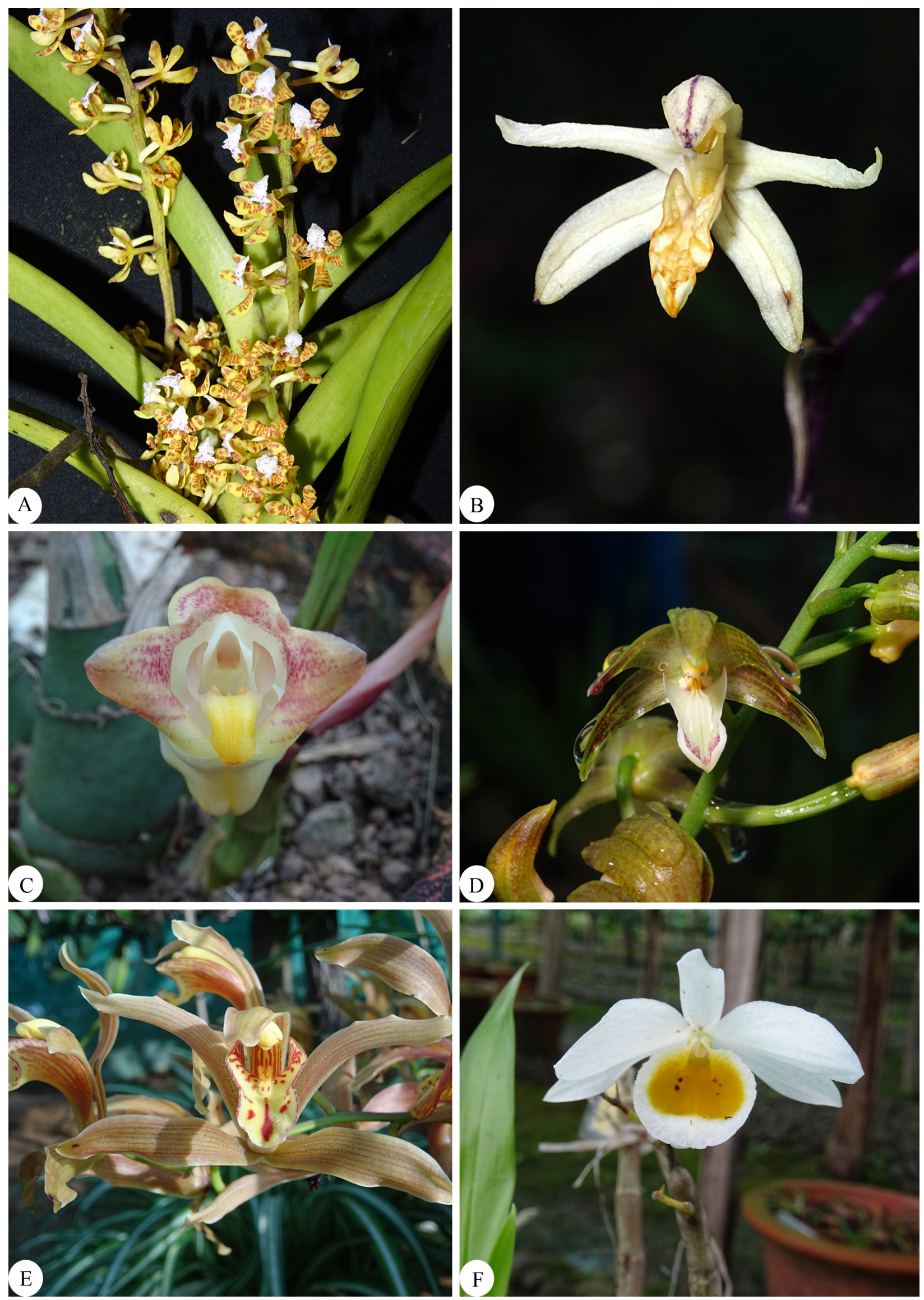

Fig. 2. A - Acampe ochracea (Lindl.) Hochr.; B - Aphyllorchis montana Rchb. f.; C - Acanthophippium sylhetense Lindl.; D - Chrysoglossum ornatum Blume; E - Cymbidium concinnum Liu et Chen; F - Dendrobium gratiotissimum Rchb. f. 


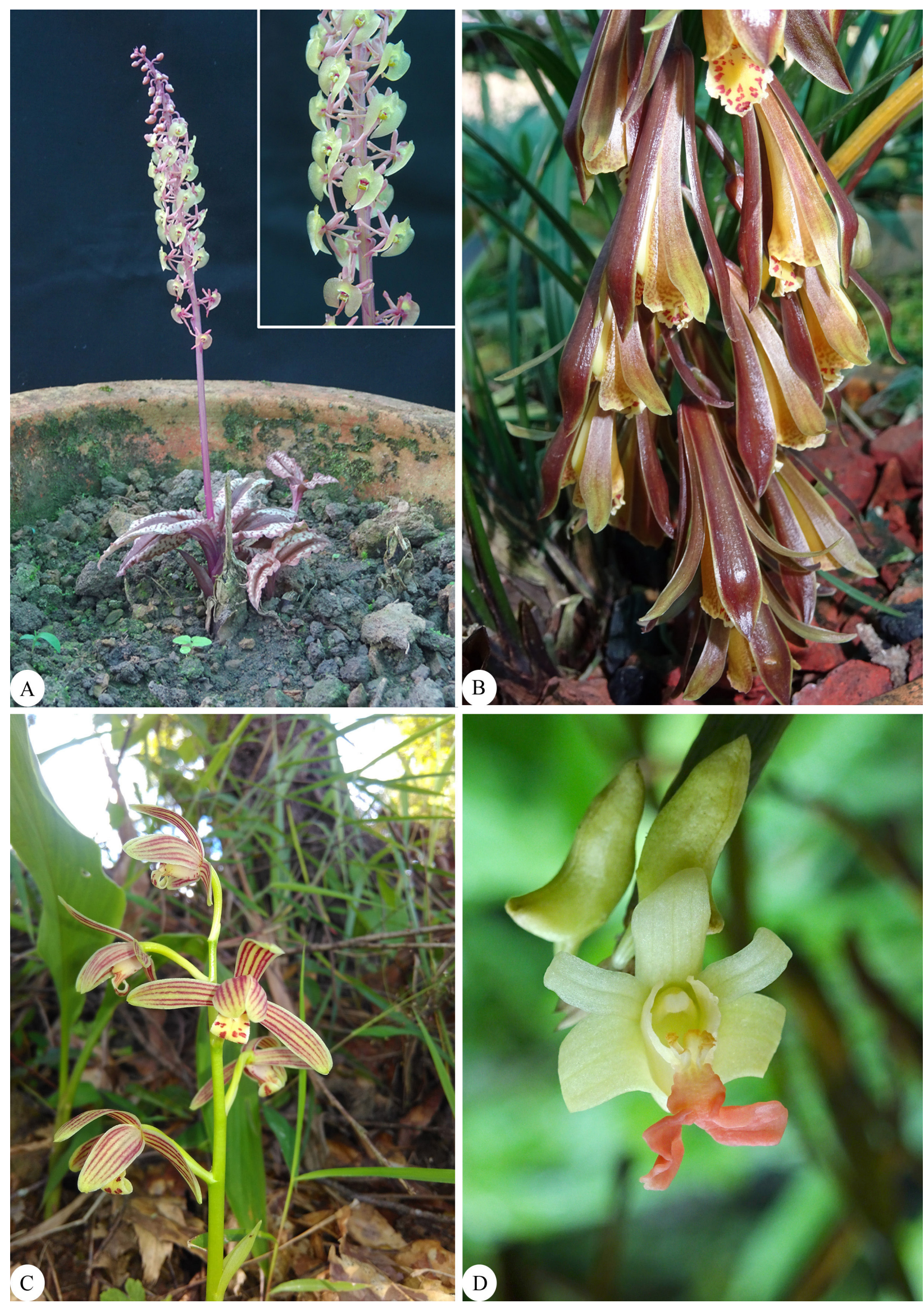

Fig. 3. A - Crepidium calophyllum (Rchb. f.) Szlach.; B - Cymbidium cochleare Lindl.; C - Cymbidium nanulum Wu et S. C. Chen; D - Dendrobium abhaycharanii (Phukan et A. A. Mao) Schuit. et P. B. Adams. 

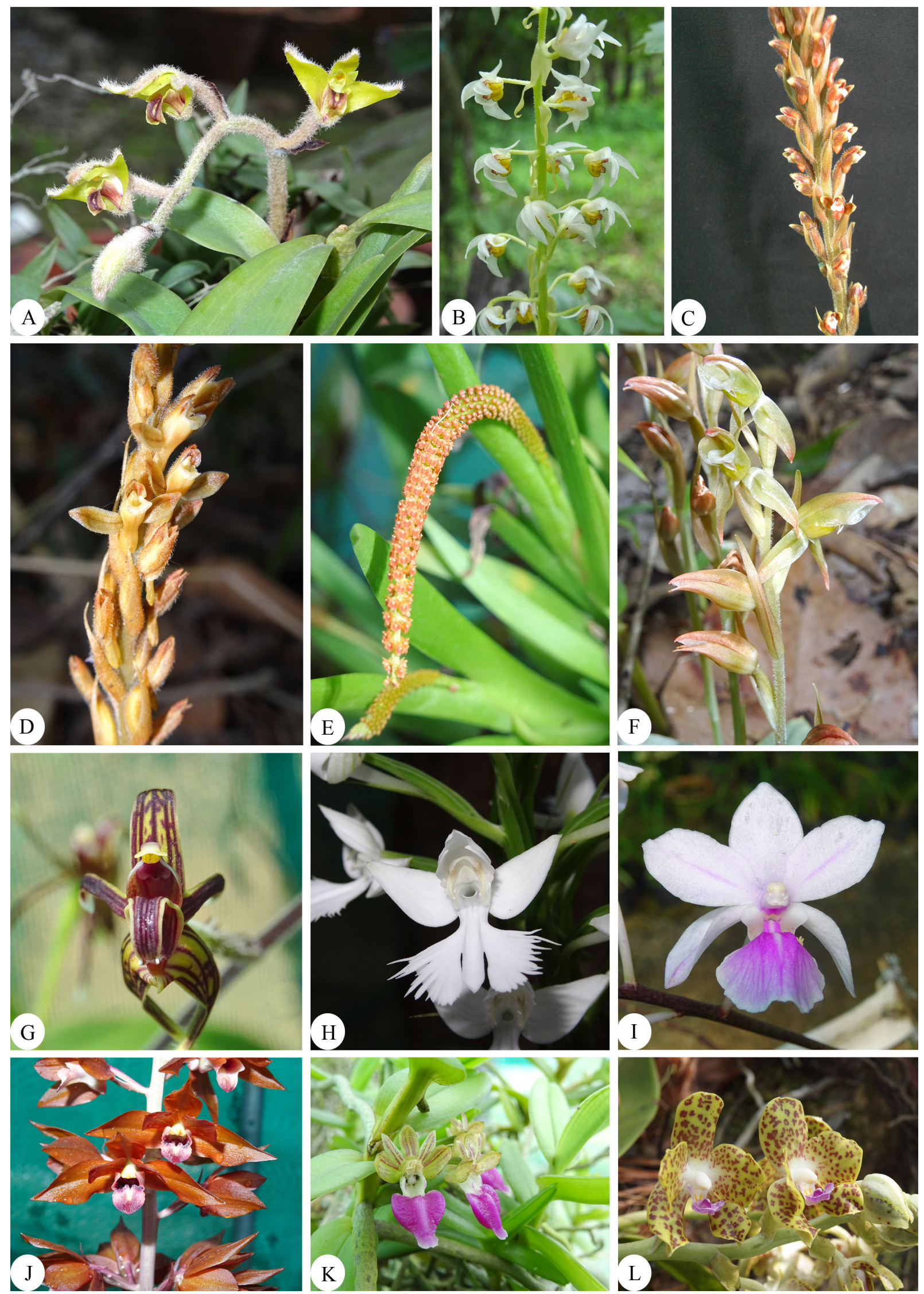

Fig. 4. A - Eria lasiopetala (Willd.) Ormerod; B - Eria sutepensis Downie; C - Erythrodes blumei (Lindl.) Schltr.; D - Erythrodes hirsuta (Griff.) Ormerod; E - Oberonia jenkinsiana Lindl.; F - Goodyera viridiflora (Blume) Dietrich; G - Eriodes barbata (Lindl.) Rolfe; H - Habenaria dentata (Sw.) Schltr.; I - Holcoglossum amesianum (Rchb. f.) Christ.; J - Eulophia zollingeri (Rchb.f.) J. J. Sm.; K - Pelatantheria insectifera (Rchb. f.) Ridl.; L - Phalaenopsis hygrochila J. M. H. Shaw. 

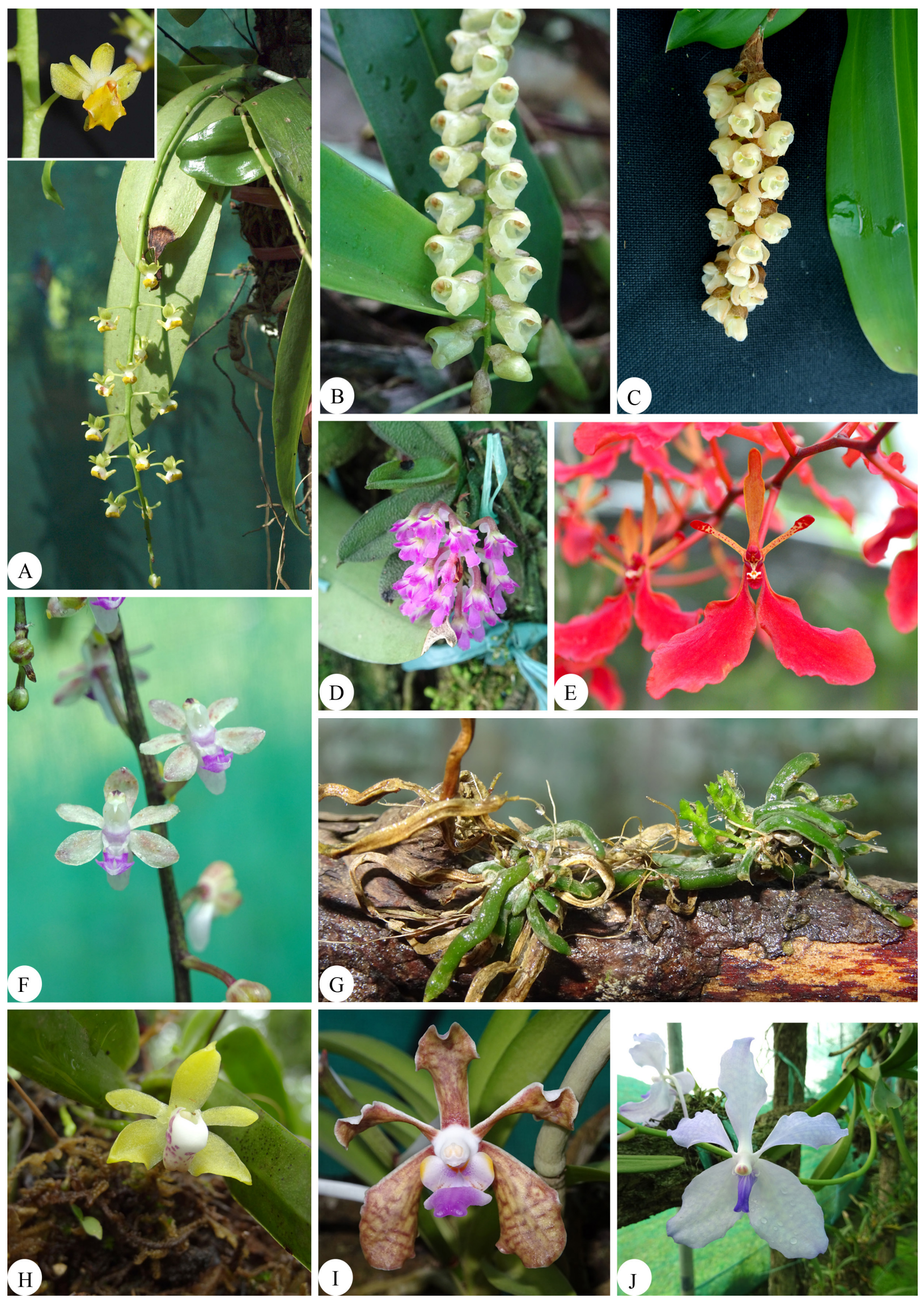

Fig. 5. A - Phalaenopsis yingjiangensis (Z. H. Tsi) Kocyan et Schuit; B - Pholidota pallida Lindl.; C - Pholidota pygmaea H. J. Chowdhery et G. D. Pal; D - Schoenorchis fragrans (C. S. P. Parish et Rchb. f.) Seidenf.; E - Renanthera imschootiana Rolfe; F - Sarcoglyphis manipurensis A. N. Rao et al.; G - Taeniophyllum glandulosum Blume; H - Thrixspermum indicum Vik. Kumar et al.; I - Vanda bicolor Griff.; J - Vanda coerulea Lindl. 


\section{Results and discussion}

Orchid Genera so far reported from Manipur are arranged in the phylogenetic classification (Chase et al., 2015). According to this classification, the 90 genera represent 4 Subfamilies, 13 Tribes and 14 Subtribes as shown below. The number of species known from Manipur and the total number of species in the genus are indicated in parentheses.

\section{Systematic overview}

Subfamily: Vanilloideae Szlach.

Tribe: Vanilleae Blume

Genera: Cyrtosia Blume (1/5), Galeola Lour. (2/6), Vanilla Mill. (1/105).

Subfamily: Cypripedioideae Kostel.

Genus: Paphiopedilum Pfitzer (5/86)

Subfamily: Orchidoideae A. A. Eaton

Tribe: Cranichideae Pfeiff.

Subtribe: Goodyerinae Klotzsch

Genera: Anoectochilus Blume (1/43), Erythrodes

Blume (2/26), Goodyera R. Br. (4/98), Myrmechis

Blume (1/17), Odontochilus Blume (2/25), Zeuxine

Lindl. (3/74).

Subtribe: Spiranthinae Lindl. ex Meisn.

Genus: Spiranthes Rich. (2/34)

Tribe: Orchideae Verm.

Subtribe: Orchidinae Verm.

Genera: Habenaria Willd. (8/835), Herminium

L. (1/19), Pecteilis Raf. (2/8), Peristylus Blume

(8/103), Ponerorchis Rchb.f. (1/55), Satyrium Sw.

$(1 / 86)$,

Subfamily: Epidendroideae Kostel.

Tribe: Neottieae Lindl.

Genera: Aphyllorchis Blume (1/22),

Cephalanthera Rich. (1/19)

Tribe: Tropidieae Dressler

Genera: Corymborkis Thouars (1/6), Tropidia Lindl. (2/31)

Tribe: Nervilieae Dressler

Subtribe: Nerviliinae Schltr.

Genus: Nervilia Gaudich. (4/67).

Tribe: Arethusea Lindl.

Subtribe: Arethusinae Benth.

Genera: Anthogonium Lindl. (1/1), Arundina

Blume (1/2)

Subtribe: Coelogyninae Benth.

Genera: Coelogyne Lindl. (21+1var./200),

Dickasonia L. O. Williams (1/1), Neogyna Rchb. f. (1/1), Otochilus Lindl. (3/5), Panisea (Lindl.) Lindl. (4/11), Pholidota Hook. (7/39), Pleione D. Don (4/21), Thunia Rchb. f. (1/5).
Tribe: Malaxideae Lindl.

Subtribe: Dendrobiinae Endl.

Genera: Bulbophyllum Thouars (39/1867),

Dendrobium Sw. (66+1var./1509).

Subtribe: Malaxidinae Benth. et Hook. f.

Genera: Crepidium Tausch (5/260), Dienia Lindl. (1/6), Liparis Rich. (11/426), Oberonia Lindl. $(14+1$ var./323)

Tribe: Cymbidieae Pfitzer

Subtribe: Cymbidiinae Benth.

Genera; Cymbidium Sw. (22/71)

Subtribe: Eulophiinae Benth.

Genera: Eulophia R. Br. (5/200), Geodorum Andrews (2/12)

Tribe: Epidendreae Lindl.

Subtribe: Calypsoinae Chase et al.

Genus: Yoania Maxim. (1/4).

Subtribe: Agrostophyllinae Szlach.

Genus: Agrostophyllum Blume (2/100)

Tribe: Collabieae Pfitzer.

Genera: Acanthephippium Endl. (1/13), Ania Lindl. (2/11), Calanthe R. Br. (7/216), Cephalantheropsis Guillaumin(2/4), Chrysoglossum Blume (1/4), Eriodes Rolfe (1/1), Nephelaphyllum Blume (1/11), Pachystoma Blume (1/3), Phaius Lour. (4/45), Spathoglottis Blume (1/48), Tainia Blume (1/23).

Tribe: Podochileae Pfitzer.

Genera: Bryobium Lindl. (1/8), Callostylis Blume (2/5), Ceratostylis Blume (1/147), Conchidium Griff. (1/10), Cryptochilus Wall. (2/5), Eria Lindl. (9/237), Mycaranthes Blume (2/36), Phreatia Lindl. (1/211), Pinalia Lindl. (6/105), Porpax Lindl. (1/13), Thelasis Blume (2/26).

Tribe: Vandeae Lindl.

Subtribe: Polystachyinae Schltr.

Genus: Polystachya Hook. (1/234).

Subtribe: Aeridinae Pfitzer.

Genera: Acampe Lindl. (2+1var./8), Aerides Lour. (3/25), Arachnis Blume (3/14), Chiloschista Lindl. (1/20), Cleisocentron Brühl (1/6), Cleisomeria G. Don (1/2), Cleisostoma Blume (9/88), Gastrochilus D. Don (5/56), Holcoglossum Schltr. (2/14), Luisia Gaudich. (4/39), Papilionanthe Schltr. (3/11), Pelatantheria Ridl. (1/8), Phalaenopsis Blume (7/70), Pteroceras Hassk. (1/27), Renanthera Lour. (1/20), Rhynchostylis Blume (2/3), Sarcoglyphis Garay. (1/12), Schoenorchis Reinw. (2/25), Smitinandia Holttum (1/3), Taeniophyllum Blume (1/185), Thrixspermum Lour. (2/161), Uncifera Lindl. (2/6), Vanda R. Br. (10/73), Vandopsis Pfitzer $(1 / 4)$. 
It may be noticed that the orchid flora of Manipur comprises of 389 taxa (including 5 varieties) belonging to 90 genera. Out of 389 taxa, there are 286 (including 3 var.) epiphytes of 49 genera, 95 (including 2 varieties) terrestrials of 38 genera, 8 mycoheterotrophs of 6 genera. It may be mentioned that some of the epiphytes like Cymbidium tigrinum, Pholidota imbricate, P. rubra etc. are found as lithophytic on moss covered rocks. There are 3 genera with species of mixed habits i. e. Cymbidium with 14 epiphytes, 6 terrestrials and 2 mycoheterotrophs; Liparis with 11 epiphytes and 1 terrestrial and Eulophia with 4 terrestrials and 1 mycotrophic species (Table).

Among larger epiphytic genera, Dendrobium tops the list with 67 species and 1 variety followed by Bulbophyllum (39), Coelogyne (21+1var.), Oberonia (14+1var.), Eria (9) etc. Among the terrestrials, Peristylus (8 species) is the largest followedby Calanthe (8), Habenaria (8), Paphiopedilum (5) etc. Among the 6 mycotrophic genera, Galeola and Cymbidium arewith 2 species each followed by Aphyllorchis, Cyrtosia, Eulophia and Yoania with one species each. There are 4 monotypic genera viz. Anthogonium, Dickasonia, Eriodes and Neogyna reported so far from the state.

\section{New records for the state}

Out of 389 orchids reported so far from Manipur, seven species viz. Bulbophyllum cornutum (Blume) Rchb. f.; Eria tomentosa (J. Koenig) Hook. f.; Liparis duthiei Hook. f.; Luisia trichorhiza (Hook.) Blume, Panisea apiculata Lindl., Pholidota rubra Lindl. and Pteroceras teres (Blume) Holtt. have been reported from Manipur for the first time as new records to the state.

\section{Threats and conservation measures}

Majority of the orchid species of Manipur are facing a serious threat of extinction in the state mainly due to their natural habitat loss caused by forest clearance for 'Jhum' cultivation or 'shifting cultivation' which is still prevalent among indigenous tribes of N. E. India. It involves slash and burning of certain area of forest to grow required agriculture crop and after harvesting the crop, that area will be abandoned for certain period of cycle. Thus, they keep on moving to different forest places for this type of cultivation and return to the first place after 5-10 years of cycle depending upon their population. Clearence of forest area for developing new townships, construction of roads and dams for generation of hydro-electricity are also playing considerable role in depletion of wild orchid populations in the state. Further, it has been noticed that ornamental orchids like Dendrobium densiflorum, D. chrysathum, Renanthera imschootiana, Vanda coerulea etc. and the species of medicinal importance like Dendrobium aphyllum, $D$. denudans, $D$. devonianum, $D$. nobile, D. fugax etc. are collected indiscriminately by the local people from the wild on large scale for commercial purpose in the domestic as well as international market. The stems of medicinal orchids are cut into pieces and made into small bundles and smuggled illegally to China via Myanmar at the cost of Rs. 500-1000 (USD 6.99 to 13.99) per kilogram.

It is suggested to bring more amount of orchid rich-forest area of the state under protected area by the state forest department with stringent rules and regulations to preserve the flora in situ on one hand, and on the other to check illegal collections from the wild. Wherever, the forest area is owned by the local tribes, those people are to be made aware of the importance of the plants occur in their forest area and their sustainable utilization. Establishment of tissue-culture laboratories in the state should be encouraged by the government to propagate many rare ornamental and medicinal plants on large scale and make available to local farmers and business people which would surely reduce the pressure on wild populations.

\section{Acknowledgements}

Sincere thanks are due to Mr. H. Kipgen, President, FEEDS, Hengbung for support and facilities; and to the SEED \& KIRAN Division, Deapartment of Science and Technology, Government of India, N. Delhi for financial assistance. Second author extends sincere thanks also to the Director, Botanical Survey of India, Kolkata for encouragemnt and to Santanu Dey for providing relevent literature.

\section{REFERENCES}

Chase M. W., Cameron K. M., Freudenstein J. V., Pridgeon A. M., Salazar G., Berg C. V. D., Schuiteman A. 2015. An updated classification of Orchidaceae. Botanical Journal of the Linnean Society 177: 151-174.

Chauhan A. S. 2001. A conspectus of orchids of Manipur: Their status and conservation. In: Orchids Science and Commerce. Eds. P. Pathak, R.N. Sehgal, N. Shekar, M. Sharma, A. Sood. Bishen Singh mahendra Pal Singh, Dehra Dun, 81-99 pp. 
Choltco T. 2009. Vanda motesiana for Martin. Orchid Review 117: 148-149.

Chowdhery H. J. 2009. Orchid diversity in North-Eastern states of India. The Journal of the Orchid Society of India 23(1-2): 19-42.

Chowlu K. 2014. A note on the validity of Bulbophyllum manipurense Satish and Suresh (Orchideaceae). National Academy Science Letters 37 (6): 513-515. DOI: 10.1007/s40009-014-0269-9

Chowlu K. 2016. Erratum to Oberonia manipurensis sp. nov. (Orchidaceae) from Manipur, India. Nordic Journal of Botany 34: 42-44. DOI: 10.1111/njb.00591

Chowlu K., Angela N., Rao A. N., Vij S. P. 2012a. Two new additions to the orchid flora of Manipur, India. The Journal of the Orchid Society of India 26(1-2): 89-92.

Chowlu K., Angela N., Rao A. N., Vij S. P. 2013a. Coelogyne fuscescens var. brunnea (Lindley) Lindley (Orchidaceae) - a new record to Manipur, India. Pleione 7(1): 275-278.

Chowlu K., Devi Y. N., Rao A. N., Angela N., Sharma H. B., Akimpou G. 2015a. Oberonia manipurensis sp. nov. (Orchidaceae) from Manipur, India. Nordic Journal of Botany 33(1): 42-44.

Chowlu K., Mahar K. S., Sharma H. B., Ngapui R., Rao A. N., Vij S. P. 2014a. Bulbophyllum dickasonii Seidenf. (Orchidaceae): a new record for India with a complete morphological description. Kew Bulletin 69(2): 9495. DOI: 10.1007/S12225-014-9495-Z

Chowlu K., Nanda Y., Rao A. N. 2014b. Oberonia acaulis Griff. var. latipetala (Orchidaceae) - a new variety from Manipur, India. Bangladesh Journal of Plant Taxonomy 21(1): 93-95. DOI: 10.3329/bjpt.v21i1.19273

Chowlu K., Nanda Y., Rao A. N., Vij S. P. 2013b. Oberonia integerrima, an addition to the Orchid flora of India. Nelumbo 55: 1-3.

Chowlu K., Nanda Y., Rao A. N., Vij S. P. 2014c. Two new additions of Peristylus B1. to the orchid flora of Manipur, India. International Journal of Scientific and Research Publications 4(3): 1-4.

Chowlu K., Nanda Y., Sharma H. B., Rao A. N. 2015b. Extended distribution of Cymbidium concinnum (Orchidaceae) and its conservation status in India. Richardiana 15: 142-150.

Chowlu K., Nanda Y., Sharma H. B., Rao A. N., Vij S. P. 2013c. Addition of two species of Sunipia (Orchidaceae) to the flora of Manipur, India. Pleione 7(2): 549-553.

Chowlu K., Nanda Y., Thithila K. S., Rao A. N. 2013d. Two new additions to the orchid flora of Manipur, India. The Journal of the Orchid Society of India 27(1-2): 89-92.

Chowlu K., Rao A. N. 2015a. Four new records of Bulbophyllum (Orchideaceae) for Manipur, India. Richardiana 15: 151-160.

Chowlu K., Rao A. N. 2015b. Synopsis of Pholidota Lindley ex W. J. Hooker (Orchidaceae) in Manipur, India with a newly recorded species. Richardiana 16: 4-17.

Chowlu K., Rao A. N., Angela N., Vij S. P. 2013e. A brief account of the genus Bulbophyllum (Orchidaceae) in Manipur. Rheedea 23(2): 86-97.

Chowlu K., Rao A. N., Vij S. P. 2012b. Oberonia jenkinsiana Griffith ex Lindley (Orchidaceae) - an addition to the flora of Manipur, India. Pleione 6(2): 406-408.

Chowlu K., Sharma H. B., Rao A. N. 2015c. Extended distribution of three Bulbophyllum species (Orchideaceae) in India and some observations about their foliar anatomy. Richardiana 15: 168-182.

Clarke C. B. 1889. On the plants of Kohima and Muneypore. The Journal of Linnean Society 25: 71-74.

Deb D. B. 1956. Floristic study of Manipur. Thesis for D. Phil. Calcutta University.

Deb D.B. 1957. Studies on the flora of Manipur. Bulletin of the Botanical Society of Bengal, Calcutta 11: 15-24.

Deb D. B. 1961. Monocotyledonous plants of Manipur Territory. The Bulletin of the Botanical Survey of India 3 : 126-129.

Deori C. 2007. Eco-Taxonomy of the genus Dendrobium Sw. (Orchidaceae) of Northeast India. Ph. D. Thesis submitted to Gauhati University, Guwahati, Assam (Unpublished).

Devi R. K. J., Ghatak J. 1984. A preliminary study on orchids of Manipur. Proc. $5^{\text {th }}$ ASEAN Orchid Congress. Singapore, 72-78 pp.

Devi Th S., Singh P. D., Somkuwar B. G., Thorat S. S., Kumar S. 2018. Oberonia pachyrachys (Orchidaceae): a new addition to the flora of Manipur, India. Jardin Botanique de Guyane 2: 1-7.

Ghatak J., Devi R. K. J. 1986. Orchids of Manipur. In: Biology, Conservation and Culture of Orchids. Ed. S. P. Vij. Affiliated East West Press Pvt. Ltd., New Delhi, 357-362 pp.

Govaerts R., Bernet P., Kratochvil K., Gerlach G., Carr G., Alrich P., Pridgeon A. M., Pfahl J., Campacci M. A., Baptista H. D., Tigges H., Shaw J., Cribb P., George A., Kreuz K., Wood J. 2018. World checklist of Orchidaceae. Facilitated by the Royal Botanic Gardens, Kew. Published on the Internet. URL: http://wcsp.science.kew.org/ (Accessed 27 June 2018).

Hooker J. D. 1888-1890. Orchidaceae. In: The Flora of British India. Ashford, Kent. Vol. 5. 667-858 pp. Vol. 6. $1-198$ pp.

Khuraijam J. S., Roy R. K. 2016. Dendrobium kentrophyllum (Orchidaceae) - a new record for the orchid flora of Manipur, India. Richardiana 17: 273-276. 
Kingdon-Ward F. 1952. Plant Hunter in Manipur. Jonathan Cape, London, 254 pp.

Kishor R., Chowlu K., Vij S. P. 2012. Ione kipgenii (Orchidaceae), a new species from Manipur, India. Kew Bulletin 67: 517-519.

Kishor R., Devi Y. N., Sharma H. B., Tongbram J., Vij S. P. 2014. Dendrobium tamenglongense sp. nov. (Orchidaceae) from Manipur, India. Nordic Journal of Botany 32(2): 150-153. DOI: 10.1111/j.1756-1051.2013.00124

Kumar C. S., Kumar P. C. S. 2005. An Orchid Digest of Manipur, Northeastern India. Rheedea 15(1): 1-70.

Kumar S., Singh, P. D., Devi H. S., Thongam B., Somkuwar B. P., Thorat S. S. 2018. Cymbidium dayanum and Cymbidium sinense (Orchidaceae): two new additions to the orchid wealth of Manipur, India. Richardiana 2: 82-87.

Kumar V. 2017. Cymbidium nanulum Y.S. Wu \& S.C. Chen, a new addition to the flora of India. Telopia 20: $137-141$.

Kumar V., Rao A. N. 2015. Goodyera viridiflora (Blume) Lindley ex D. Dietrich (Orchidaceae): a new distributional record for Manipur, India. The MIOS Journal 16 (9): 4-8.

Kumar V., Rao A. N., Agrawala D. K. 2015. Tropidia namasiae (Orchidaceae): a new distributional record for India from Manipur. Richardiana 15: 317-322.

Kumar V., Sharma H. B., Rao A. N. 2016a. Sarcoglyphis manipurensis sp. nov. (Orchidaceae) from Manipur, India. Nordic Journal of Botany 34: 191-196.

Kumar V., Sharma H. B., Rao A. N. 2016b. Two new additions of orchids to the flora of Manipur (India). NeBIO 7(3): 66-70.

Kumar V., Verma D., Rao A. N. 2017. Thrixspermum indicum (Orchidaceae), a new species from Northeast India. Phytotaxa 292(1): 79-84.

Laiphrakpam L., Ghosh S. K., Das A. K., Singh P. K. 2014. Conservation of Orchids in Imphal Valley, Manipur. International Journal for Innovative Research in Science and Technology 1(1): 11-13.

Laiphrakpam L., Ghosh S. K., Das A. K., Singh P. K. 2015. Orchidaceae family in Imphal East, Manipur. International Journal for Innovative Research in Science and Technology 1(9): 183-185.

Mao A.A. 1999. Notes on the orchids of Senapati and surrounding hills in the state of Manipur, India. The Journal of the Orchid Society of India 13(1-2): 55-58.

Meitei L. R., Rabha N. N. Deori C., Mao A. A. 2014. A new generic record and distribution notes on two orchid species from Manipur, India. Richardiana 14: 274-279.

Mukerjee S. K. 1953. An enumeration of the orchids of Ukhrul, Manipur. Notes from the Royal Botanic Garden, Edinburgh 21: 149-154.

Nanda Y., Chowlu K., Akimpou G., Rao A. N. 2014a. Eulophia zollingerii (Rchb. f.) J. J. Sm. and Pelatantheria insectifera (Rchb. f.) Ridl. - two new additions to the orchid flora of Manipur. Nelumbo 56: 248-251.

Nanda Y., Chowlu K., Rao A. N. 2014b. Dendrobium hesperis (Seidenf.) Schuiteman \& Adams - a new orchid record to North-East India from Manipur, India. Pleione 8(2): 523- 525.

Nanda Y., Chowlu K., Rao A. N. 2014c. Four new additions of orchids to the orchid flora of Manipur, India. NeBIO 5(6): 25-30.

Nanda Y., Chowlu K., Rao A. N. 2014d. Four new records of orchids to Manipur. NeBIO5(1): 11-17.

Nanda Y, Chowlu K., Rao A. N., Vij S. P. 2013a. Two new records of Eria Lindl. (Orchidaceae) to Manipur (India). NeBIO 4 (5): 89-90.

Nanda Y., Chowlu K., Sharma H. B., Rao A. N., Vij. S. P. 2013b. Four new records of Dendrobium (Orchidaceae) to Manipur (India). Nelumbo 55: 102-108.

Nanda Y., Kishor R. K., Vij S. P. 2012a. Taeniophyllum glandulosum B1. A new addition to Orchid flora of Manipur. The journal of the Orchid Society of India 26(1-2): 49-51.

Nanda Y., Sharma H. B., Kishor R. K., Rao A. N., VijS. P. 2012b. Dendrobium bicameratum Lindley (Orchidaceae)an addition to the flora of Manipur, India. Pleione 6(2): 450-452.

Nanda Y., Sharma H. B., Rao A. N., Vij S. P. 2013c. Contributions to the Orchid flora of Manipur (India) - 1. Pleione 7(2): 560-566.

Phukan S. J. 1996. Vanda liouvillei Finet - A new record from India. Orchid Review 104: 189-190.

Phukan S. J., Mao A. A. 2002a. Additions to the Indian Orchid Flora. Orchid Review 110: 157-161.

Phukan S. J., Mao A. A. 2002b. Armodorum senapatianum - a new species from India. Orchid Review 110: 298-300.

Phukan S. J., Mao A. A. 2004. Additions to the Indian Orchid Flora. Orchid Review 112: 115-118.

Pradhan U. C. 1976. Indian Orchids: Guide to Identification and Culture. Vol. 1. Calcutta.

Pradhan U. C. 1979. Indian Orchids: Guide to Identification and Culture. Vol. 2. Kalimpong.

Ramakantha $\boldsymbol{V}$. 1995. A report on the threatened orchids of Manipur. The journal of the Bombay Natural History Society 92: 144-145.

Rao A. N. 2007. Orchid flora of North East India- an upto date analysis. Bulletin of Arunachal Forest Research 23(1\& 2): 6-38. 
Rao A. N. 2015. Project Completion progress report of Centre for Orchid Gene Conservation of Eastern Himalayan Region (COGCEHR) submitted to SERB, department of Science and Technology, Government of India, N. Delhi, $1-222 \mathrm{pp}$.

Rao A. N., Chowlu K., Kumar V., Sharma H. B., Devi Y. N. 2016. Orchids of North East India. Vol. 1. FEEDS, Hengbung, Manipur, India.

Rao A. N., Kumar V. 2015. Record of holomycotrophic Aphyllorchis montana H. G. Reichenbach (Orchidaceae) from Manipur, India. Pleoine 9(2): 527-530.

Rao A. S., Hajra P. K. 1974. Eulophia mannii Hook. f. - a scarcely known ground orchid from Assam. The Bulletin of the Botanical Survey of India 16(1-4): 156-157.

Sharma H. B., Rao A. N., Bhattacharyya D. 2015. Dendrobium cumulatum (Orchidaceae). An addition to the orchid flora of Manipur, India. Richardiana 16: 60-64.

Shukla U., Baishya A. K. 1979. A contribution to the flora of Manipur. Journal of Bombay Natural History Society 76: 224-229.

Singh E. J., Singh N. K. S., Singh N. R. 2010. Biodiversity conservation and natural resources in North East India with special reference to Manipur. NeBIO 1(1): 42-47.

Surveswaran S., Kumar P., Sun M. 2017. Spiranthes himalayensis (Orchidaceae, Orchidoideae) a new species from Asia. PhytoKeys 89: 115-128.

Watt G. 1888. The forests of Manipur. Indian Forester 14: 291-299, 338-344, 387-394.

Yumkham S. D., Chakpram L., Benniamin A., Singh P. K. 2013. Discovery of Cyrtosia nana (Orchidaceae) in India Taiwania 58(3): 229-232. 